\title{
Controlling the Aqueous Solubility of PNIPAM with Hydrophobic Molecular Units
}

\author{
Rahul Singh ${ }^{1}$, Sanket A. Deshmukh ${ }^{2}$, , Ganesh Kamath ${ }^{2}$, Subramanian K.R.S. \\ Sankaranarayanan $^{2}$ and Ganesh Balasubramanian ${ }^{1, \%}$ \\ ${ }^{1}$ Department of Mechanical Engineering, Iowa State University, Ames, IA, USA \\ ${ }^{2}$ Center for Nanoscale Materials, Argonne National Laboratory, Argonne, IL, USA
}

\begin{abstract}
The effect of co-polymerization of thermo-sensitive polymers with hydrophilic or hydrophobic co-monomers on the LCST and the structure of proximal water remains a fundamental and challenging problem. Here, we employ all-atom molecular dynamics simulations to investigate the aqueous solubility of a thermo-sensitive polymer, poly(N-isopropylacrylamide) (PNIPAM), a hydrophobic polymer polystyrene (PS) and block co-polymers of PNIPAM-co-PS. The simulations of pure oligomers of PNIPAM and PS and their co-polymers are conducted below and above the LCST of PNIPAM to elucidate the effect of increase in number of PS units in PNIPAM-co-PS co-polymers on the coil-to-globule transition of PNIPAM and structure of proximal water. Our simulations suggest that while the LCST of pure PNIPAM oligomers is strongly associated with the structural changes in the proximal water molecules present near PNIPAM, inclusion of PS units disrupts the interactions between PNIPAM and water and promotes the faster dehydration of PNIPAM chains above its LCST. This phenomenon is manifested by a coil-to-globule transition in PNIPAM chains present in PNIPAM-co-PS copolymers at much shorter times as compared to the pure PNIPAM oligomers. Our results also reveal that with increase in the number of PS units in PNIPAM-co-PS co-polymers the coil-toglobule

transition

is

accelerated.
\end{abstract}

${ }^{*}$ Corresponding author: bganesh@iastate.edu

Address: 2092 Black Engineering Building, Iowa State University, Ames, IA 50011, USA

" Corresponding author: sanket@anl.gov

Address: Center for Nanoscale Materials, Argonne National Laboratory, Lemont, IL 60439, USA 


\section{I) Introduction}

Poly(N-isopropylacrylamide) (PNIPAM) is a classic thermo-sensitive polymer that was first synthesized in the 1950s and is still one of the most commonly studied and utilized intelligent polymers. ${ }^{1}$ PNIPAM has a lower critical solution temperature (LCST) around $305 \mathrm{~K}$ in water and exhibits a coil-to-globule transition at temperatures above its LCST. The hydrogen-bonding interactions between the hydrophilic groups of PNIPAM and water are dominant below the LCST, enforcing a coil-like conformation in PNIPAM. ${ }^{2}$ However, when the temperature is raised above the LCST, the solvation entropy of the hydrophobic groups in the backbone and side-chain of PNIPAM dominates and leads to a globule-like structure of PNIPAM in aqueous solution. In particular, above the LCST the hydrophobic backbone and isopropyl groups in the side-chain tend to associate, which causes the collapse of PNIPAM chains and intermolecular aggregation. ${ }^{3}$

PNIPAM, its architectures (hydrogels, brush structures etc.), and co-polymers have been widely investigated experimentally and computationally. ${ }^{2,4-15}$ The focus of most of the computational studies has been on exploring the structure of PNIPAM under different environmental conditions. ${ }^{11,16-18}$ The variation in LCST of PNIPAM with force fields, ${ }^{11}$ ions in the solution, ${ }^{19}$ and different chain lengths ${ }^{11,16,18}$ have been examined using all-atom and united atom molecular dynamics (MD) simulations. Previously, we have performed MD simulations of NIPAM monomers using different force-fields (namely AMBER, OPLS-AA, CHARMM and GROMOS $)^{20}$ and their suitability to predict different thermodynamic properties. All the force fields showed a decrease in free energy of hydration across LCST, thus proving that NIPAM becomes hydrophobic as the temperature is raised above its LCST. We have also investigated the effect of PNIPAM chain length on its LCST. ${ }^{2}$ The results showed that PNIPAM with 30 monomer units (30-mer) or more undergo a coil-to-globule transition above its LCST while for short oligomer chains (3-, 5-, and 10-mers), this transition is not observed. Longhi et al. studied PNIPAM oligomers consisting of 50 monomers at $300 \mathrm{~K}$ and at $310 \mathrm{~K}$ (i.e., below and above the LCST), ${ }^{16}$ and observed a coil-to-globule transition and reduced number of water molecules in the first coordinate shell of PNIPAM at $310 \mathrm{~K}$ as compared to $300 \mathrm{~K}$ suggesting different arrangement of hydrophobic groups at the two temperatures. ${ }^{16}$ There are several experimental and computational investigations of PNIPAM based architectures such as hydrogels ${ }^{4,6,21,22}$ and 
brushes ${ }^{23}$ including our previous work on the effect of cross-linker length on the structural and dynamical properties of PNIPAM hydrogels across its LCST. ${ }^{20}$ Our results revealed that the cross-linker length did not affect the temperature of the onset of the LCST, although the degree of swelling and pore size distribution in these hydrogels were strongly dependent on the length of cross-linkers.

The LCST of PNIPAM is around the human body temperature and can be altered by copolymerization of hydrophilic and/or hydrophobic monomers with PNIPAM ${ }^{24-26}$ offering the possibility to design PNIPAM based materials suitable for a range of biomedical applications. ${ }^{27-}$ ${ }^{29}$ Additionally, the control of polymer properties through the synthesis of block co-polymers to create multifunctional complex macromolecules is potentially applicable in energy materials, cosmetics, medical diagnostics, and biotechnology. ${ }^{30,31}$ Recently, MD simulations have been used to investigate the coil-to-globule transition in co-polymers of PNIPAM with poly(ethylene glycol) methacrylate (PEGMA) (PNIPAM-co-PEGMA) in $1 \mathrm{M} \mathrm{NaCl}$ solution. ${ }^{32}$ The PNIPAMco-PEGMA is observed to go through the hydrophilic to hydrophobic conformational change at a temperature of $318 \mathrm{~K}$, slightly above the LCST of PNIPAM. These simulations of PNIPAM-coPEGMA showed a large increase in hydrodynamic radius and aggregate molar mass above the LCST. On the other hand, experiments have shown that the presence of polystyrene (PS), a nonthermo-sensitive hydrophobic polymer, lowers the LCST of PNIPAM. ${ }^{31,33-35}$ Increase in the concentration of such hydrophobic moieties can result in PNIPAM co-polymers with water insoluble properties. ${ }^{36-38}$ Thus, these block co-polymers can combine the characteristics of each block, creating a new polymer with unique capabilities. However, one of the biggest obstacles in controlling the macroscopic properties of these co-polymers is the lack of understanding of their atomic-level structure and network of proximal solvent molecules. Even with the advances in experimental techniques it is almost impossible to probe the dynamics of atomic-level structure of these co-polymers and proximal water. In addition to the exact atomic origin of the LCST, the effect of the nature (hydrophilic vs. hydrophobic) of the co-monomer and chain length of the copolymer on the LCST of PNIPAM is unknown. Structure of water at the junction (where two polymers are attached) of these co-polymers is inaccessible by experiments.

Another important parameter is the tacticity of the polymer. For most of the polymers their tacticity is known to have an effect on the polymer chain conformation and dynamics. Several 
computational and experimental reports suggest that the tacticity of PNIPAM affects its responsiveness to aqueous media. ${ }^{39-42}$ For example, Ray et al. show that the LCST of PNIPAM decreases with increase in meso diad $(\mathrm{m})$ content. They found that PNIPAM chains with $\mathrm{m}$ of 45 and $66 \%$ exhibit LCST at 31.1 and $17{ }^{\circ} \mathrm{C}$, respectively. A recent computational study conducted by Chiessi and Paradossi was focused on PNIPAM 30-mers with content of meso diads, m, of $45 \%$ and 59\%. Their study revealed that below its LCST number of hydrogen bonds per water molecule in the first hydration shell and the water surface concentration around PNIPAM are affected by the meso diad ratio. This results in the higher hydrophobicity of the isotactic-rich PNIPAM chain. On the other hand, above the LCST the subtle effects leading to the modulation of conformation and hydration by means of tacticity are overtaken, and the chain collapse is observed for both systems, with of 45 and $59 \%$, up to a similar globular state.

Here, we investigate block co-polymers of PNIPAM-co-PS to explore the effect of increase in hydrophobicity of PNIPAM on its coil-to-globule transition. We employ all-atom MD simulations to examine the role of PS units on the LCST predictions of PNIPAM. Simulations of PNIPAM-co-PS with varying number of segments of PS monomer units are carried out below and above the LCST (at $275 \mathrm{~K}$ and $325 \mathrm{~K}$ ). Simulation trajectories are analyzed to calculate radius of gyration $\left(\mathrm{R}_{\mathrm{g}}\right)$, small angle neutron scattering spectra (SANS), the distribution of PS units near a PNIPAM oligomer chain, and radial distribution function (RDF) to identify the nature of conformational transition of PNIPAM in PNIPAM-co-PS co-polymers. Dynamic evolution of structure of water is examined by inspecting the first hydration shell of co-polymer chains during simulations. The strength of polymer-water interactions is scrutinized by calculating residence time probability $\left(\mathrm{P}_{\text {res }}(\mathrm{t})\right)$ of water molecules near the polymer chains. The hydrogen bonding characteristics between the polymer and water are evaluated to determine the effect of temperature and LCST transition on the structure of proximal water. Where possible, simulation results are compared both qualitatively and quantitatively with existing experimental and computational studies.

\section{II) Methods}

\section{A) Computational Procedures and Simulation Details}


All-atom MD computations are performed using Nanoscale Molecular Dynamics (NAMD) ${ }^{44}$ a highly parallelized code for large-scale simulations. The CHARMM36 (Chemistry at HARvard Macromolecular Mechanics) ${ }^{45}$ force field is used together with the modified TIP3P water model for all the simulations. CHARMM36 force field has been successfully employed to study PNIPAM and other polymers. ${ }^{20,46,47} \mathrm{We}$ explore the effect of increase in the hydrophobicity of PNIPAM on its LCST behavior using atomistic models of PNIPAM-co-PS with varying content of PS monomer units as described in Table 1 and Figure 1. The structure generation method for all the polymer models is adopted from our previous work. ${ }^{2}$ Based on the CHARMM36 force field, the distance between carbon atom of backbone $-\mathrm{CH}_{2}-$ group of the first monomer and the carbon atom of backbone $-\mathrm{CH}-$ group of the second monomer is chosen to be $\sim 1.538 \AA$. The second monomer is rotated randomly along the axis of the backbone of the polymer chain, i.e. in $x$-direction. This method of addition of a monomer unit to a polymer chain is followed to generate a polymer chain with desired numbers of monomer units. In the case of PNIPAM-co-PS co-polymers, we generate individual structures of PNIPAM and PS polymers. This is followed by removal of a hydrogen atom on the carbon atom present at the end of both PNIPAM and PS oligomer chains. After constructing the primary structure, the two chains are then placed in a simulation cell such that the two hydrogen deficient carbon atoms on each chain are within $\sim 1.6$ $\AA$ from each other. A bond is created between these two chains and the polymer chains are relaxed for $200 \mathrm{ps}$ in vacuum.

As mentioned earlier, several computational and experimental reports suggest that the tacticity of PNIPAM affects its responsiveness to aqueous media. ${ }^{39-42}$ Pure PS chains, regardless of their tacticity, are insoluble in water. However, when a PS chain is copolymerized with PNIPAM, the copolymer PNIPAM-co-PS is soluble in water. ${ }^{43}$ The tacticity of a PS chain might influence the configuration of PNIPAM in PNIPAM-co-PS copolymer by modifying the interactions between PNIPAM and water, thus altering the LCST of PNIPAM-co-PS copolymers. However, studying the effect of tacticity of either PS and/or PNIPAM chains on the LCST of PNIPAM is beyond the scope of this work. In the present study, our focus has been on understanding the effect of presence of PS chains on the interactions between PNIPAM and water molecules. Thus, we have generated structures of PNIPAM and PS chains with meso-diad content of $\sim 50 \%$. These polymer chains are placed in a cubic simulation box that is subsequently filled with water molecules (modified TIP3P water model) to an initial water density of $1 \mathrm{~g} / \mathrm{cm}^{3}$. The long-range 
electrostatic interactions are calculated by Particle Mesh Ewald (PME) method. The simulations are conducted under an NVT ensemble followed by NPT ensemble with the target pressure of 1 atmosphere. The Nose-Hoover thermostat is employed to constrain the temperature. The oscillation and damping time for the Langevin piston method are taken as $100 \mathrm{fs}$ and $50 \mathrm{fs}$, respectively. We use periodic boundary conditions, LJ cut-off of $12 \AA$ for all non-bonded interactions, and a time-step of $1 \mathrm{fs}$ for all simulations. The simulation temperatures are chosen to be below and above the LCST of PNIPAM, $275 \mathrm{~K}$ and $325 \mathrm{~K}$, respectively. The total simulation time is varied from $20 \mathrm{~ns}$ to $40 \mathrm{~ns}$ based on the system under consideration, as listed in Table 1 and shown via representative illustrations in Figure 1. For example, in the case of a pure chain of PS, simulations are carried out for $20 \mathrm{~ns}$ as a PS chain being hydrophobic in nature transforms and remains in a globule-like state after $12 \mathrm{~ns}$ simulation run as shown later in Figure 2. Similarly, for the systems with PNIPAM chains, simulation times are decided based on how long it takes for PNIPAM to undergo a coil-to-globule transition above its LCST. The analysis of the simulation trajectories is carried out over the last $2 \mathrm{~ns}$ of each simulation run.

Table 1: Simulation details of the molecular systems investigated.

\begin{tabular}{ccccccccc}
\hline System & $\begin{array}{c}\text { No. of } \\
\text { PNIPAM } \\
\text { monomers }\end{array}$ & $\begin{array}{c}\text { No. of PS } \\
\text { monomers }\end{array}$ & $\begin{array}{c}\text { No. of } \\
\text { water } \\
\text { molecules }\end{array}$ & $\begin{array}{c}\text { Total } \\
\text { simulation } \\
\text { time }(\mathbf{n s})\end{array}$ & $\begin{array}{c}\text { Length of a } \\
\text { side of cubic } \\
\text { simulation } \\
\text { box }(\AA)\end{array}$ & $\begin{array}{c}\text { Initial } \\
\text { Density } \\
\left(\mathbf{g} / \mathbf{c m}^{3}\right)\end{array}$ & $\begin{array}{c}\text { Final } \\
\text { Density } \\
\left(\mathbf{g} / \mathbf{c m}^{3}\right) \\
\mathbf{2 7 5 ~ K}\end{array}$ & $\begin{array}{c}\text { Final } \\
\text { Density } \\
\left(\mathbf{g} / \mathbf{c m}^{3}\right)\end{array}$ \\
\hline 325 K \\
\hline A & 30 & 0 & 16935 & 40 & 80 & 1.0 & 0.99 & 0.96 \\
B & 0 & 30 & 16865 & 20 & 80 & 1.0 & 1.02 & 1.01 \\
C & 30 & 30 & 112493 & 35 & 150 & 1.0 & 1.02 & 0.98 \\
D & 30 & 60 & 228826 & 20 & 200 & 1.0 & 1.02 & 0.99 \\
\hline
\end{tabular}


(I)

(i)
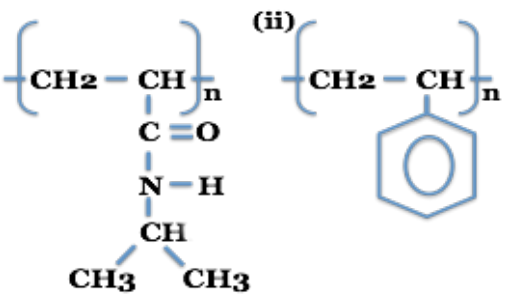

(II) ${ }_{\text {System }}$

Poly(N-isopropylacrylamide)

Polystyrene

A.

PNIPAM

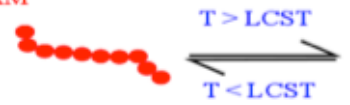

PS

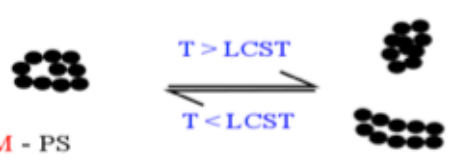

C.

PNIPAM - PS
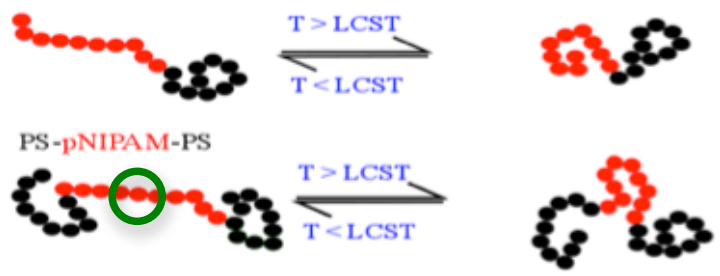

Figure 1: (I) Atomistic representation of (i) PNIPAM and (ii) PS monomer units. (II) Representative cartoons showing the structural evolution of PNIPAM and its co-polymers with PS. The illustration shows the change in the structure at temperatures below and above LCST. Green circle highlights the location of central monomer for PNIPAM.

\section{B) Analyses of the Structure and Dynamics}

We analyze the simulated trajectories to calculate radius of gyration $\left(\mathrm{R}_{\mathrm{g}}\right)$ and small angle neutron scattering (SANS) for polymer chains, distribution of PS monomers near a PNIPAM oligomer chain, radial distribution function (RDF) of key atom pairs involved in hydrogen bonding, and the numbers of water molecules within the first hydration shell of polymer chains. $\mathrm{R}_{\mathrm{g}}$ describes the polymer size and its temporal variation is used to determine the coil-like vs globule-like state of the polymer. Generally, $\mathrm{R}_{\mathrm{g}}$ provides a quantitative measure of the distribution of structure as the polymer folds. The relationship between chain length and $\mathrm{R}_{\mathrm{g}}$ can be described in the form of its square, which is the second moment around the center of mass (COM) of the chain and is defined as the mean square of the distance between the beads and the center of mass. In other words, the chain occupies a space of a sphere of radius $\mathrm{R}_{\mathrm{g}}$ with $\mathrm{COM}$ of polymer chain being the 
center of the sphere. For each polymer chain, we obtain the ensemble average for squared $\mathrm{R}_{\mathrm{g}}$ by analyzing the simulation trajectories in the $x, y$ and $z$ directions. $\mathrm{R}_{\mathrm{g}}$ is calculated over the entire simulation trajectory to show its time evolution at simulated temperatures. This approach allowed us to demonstrate a coil-to-globule transition in PNIPAM at temperatures above its LCST (at $325 \mathrm{~K}$ ) by clearly distinguishing the coil-state at the beginning of simulation and the globule-state at the end of simulation.

SANS spectra has been widely used to study biomolecules and macromolecules. ${ }^{4-52}$ In addition to the $\mathrm{R}_{\mathrm{g}}$ measurements the SANS spectra allows us to determine the shape and size of macromolecules. Here we use the CRYSON program to calculate the SANS spectra of all the polymers under investigation. Further structural changes in polymer chains are also explored by calculating the radial distribution functions (RDFs) for the polymer atoms. In addition, to examine the structure of water near polymer chains the RDFs for key atoms of polymer that are involved in hydrogen bonds with water are calculated. Number of water molecules associated with the polymer (the first hydration shell) is another measure of extent of hydration of polymer chain. We choose the cut-off distance for the first hydration shell to be $5.4 \AA$ from the surface of the polymer, based on the first minimum of the RDF between key polymer atoms involved in hydrogen bonds and water molecules.

Residence probability $\left(\mathrm{P}_{\text {res }}(\mathrm{t})\right)$ of water molecules near the hydrophilic groups of PNIPAM polymer chains is calculated both at $275 \mathrm{~K}$ and $325 \mathrm{~K} \cdot{ }^{12,53} \mathrm{P}_{\text {res }}(\mathrm{t})$ is defined as the probability of continuous existence of a water molecule near hydrophilic regions for the time interval between $t_{0}$ and $t+t_{0}$. $P_{\text {res }}(t)$ is averaged over several different simulation frames that are collected in intervals of 1 ps over a time frame of last 2 ns of simulation run. The two different regions for water molecules are defined based on the distance between water molecules and polymer chains. For example, water molecules located within $5.4 \AA$ of hydrophilic groups $(\mathrm{C}=\mathrm{O}$ and $\mathrm{N}-\mathrm{H}$ groups in side-chains of PNIPAM) are considered as hydrophilic water molecules.

In addition, simulation trajectories are also analyzed to calculate hydrogen bond correlation for hydrogen bonds formed between polymer and water. We define the hydrogen bonds between polymer and water by modifying the following geometric criteria that has been widely used to identify hydrogen bonds between water molecules: ${ }^{54}$ 


$$
\mathrm{R}_{\mathrm{OO}} \leq 3.6 \AA, \mathrm{R}_{\mathrm{OH}} \leq 2.45 \AA, \phi \leq 30^{\circ} \ldots \ldots \ldots \text { Eq. } 1
$$

$R_{O O}$ and $R_{O H}$ represent the distances between oxygen and oxygen and between oxygen and hydrogen of water molecule A and B, respectively, which are involved in hydrogen bond formation. The angle $\phi$ is between oxygen of water molecule A and oxygen \& hydrogen of water molecule $\mathrm{B}\left(\mathrm{O}_{\mathrm{A}} \cdots \cdots \mathrm{O}_{\mathrm{B}}-\mathrm{H}_{\mathrm{B}}\right)$. We have used similar method previously to study hydrogen bonding characteristics between polymer and water and for water under confinement. ${ }^{2,4,11}$

In the systems simulated, based on donor and acceptor pair concept, following hydrogen bonds are possible: A hydrogen bond between polymer (donor) and polymer (acceptor) (Type 1) and two types of hydrogen bonds are possible between polymer and water: (1) bond between hydrogen atoms attached to the nitrogen $\left(\mathrm{N}_{\mathrm{pol}}\right)$ atoms of PNIPAM (donor) and water oxygen (acceptor) $\left(\mathrm{O}_{\mathrm{w}}\right)$ (Type 2) and (2) bond between oxygen of PNIPAM ( $\left.\mathrm{O}_{\mathrm{pol}}\right)$ (acceptor) and hydrogen of water $\left(\mathrm{H}_{\mathrm{w}}\right)$ (donor) (Type 3). Similar to our previous study on polymer-water systems, based on the location of the $1^{\text {st }}$ peak of the RDF for respective donor and acceptor atoms, we define the geometric criteria for hydrogen bonds between polymer and polymer, between polymer and water, and between water and polymer. ${ }^{2,411}$ For example, to define the hydrogen bonds between polymer and water, where polymer is donor, mainly hydrogen atoms bonded to nitrogen atoms $\left(\mathrm{N}_{\mathrm{pol}}-\mathrm{H}_{\mathrm{pol}}\right)$ of PNIPAM, and oxygen of water $\left(\mathrm{O}_{\mathrm{w}}\right)$ is acceptor, the first distance criterion $\left(\mathrm{R}_{\mathrm{Npol}-\mathrm{Ow}}\right)$ requires the distance between $\mathrm{N}_{\text {pol }}$ atom $\mathrm{O}_{\mathrm{w}}$ to be $\leq 3.05 \AA$. The second distance criterion $\left(\mathrm{R}_{\mathrm{Ow}-\mathrm{Hpol}}\right)$ requires the distance between $\mathrm{O}_{\mathrm{w}}$ and $\mathrm{H}_{\mathrm{pol}}$ atom to be $\leq 2.05$ $\AA$ and the angle between acceptor-donor-hydrogen $\left(\mathrm{O}_{\mathrm{w}}-\mathrm{N}_{\mathrm{pol}}-\mathrm{H}_{\mathrm{pol}}\right)$ to be $\leq 30^{\circ}$.

$S_{i j}$, a hydrogen bond occupation number is defined to distinguish between the hydrogen bonded and non-hydrogen bonded atoms. ${ }^{2} S_{i j}=1$ for hydrogen-bonded atoms and 0 for others. A timedependent intermittent autocorrelation function, $C_{x}(t)$, of this state variable $S_{i j}$ allows us to determine the stability of the hydrogen bonds. $S_{i j}$ was allowed multiple transitions from 1 to 0 till the final breakage of hydrogen bond between atom $i$ and atom $j$ occurs. ${ }^{54}$

$$
C_{x}(t)=\left\langle\frac{\sum_{i j} S_{i j}\left(t+t_{0}\right) . S_{i j}\left(t_{0}\right)}{\sum_{i j} S_{i j}\left(t_{0}\right) \cdot S_{i j}\left(t_{0}\right)}\right\rangle
$$




\section{III) Result and Discussion}

\section{A. Conformations of Polymer Chains}

\section{(i) Radius of Gyration $\left(\mathrm{R}_{\mathrm{g}}\right)$ and end-to-end Distance}

Figures 2(a) and (b) show the radius of gyration $\left(\mathrm{R}_{\mathrm{g}}\right)$ of individual chains of PNIPAM and PS consisting of 30 monomer units (30-mer) (system A and B from Table 1), respectively. The mean value of $\mathrm{R}_{\mathrm{g}}$ has been calculated from the values of $\mathrm{R}_{\mathrm{g}}$ during the last $2 \mathrm{~ns}$ of the simulation.
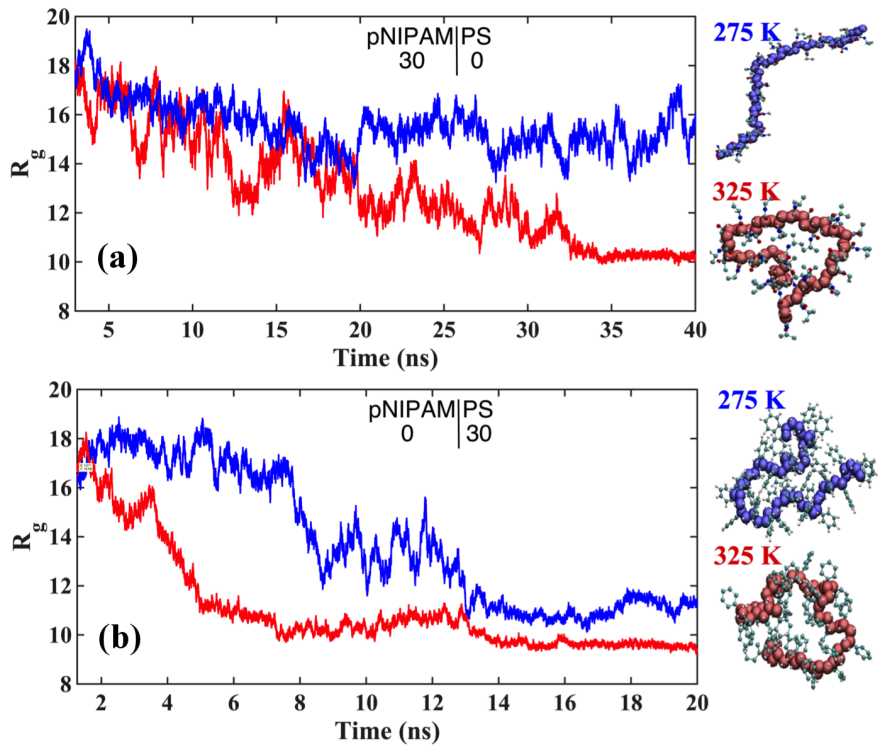

Figure 2: Radius of gyration $\left(R_{g}\right)$ and corresponding snapshots of (a) PNIPAM and (b) PS 30mer chains at $275 \mathrm{~K}$ and $325 \mathrm{~K}$. Blue and red colorations are respectively for the two temperatures $275 \mathrm{~K}$ and $325 \mathrm{~K}$. The mean value of $R_{g}$ has been sampled over the last 2 ns of the simulations.

$\mathrm{R}_{\mathrm{g}}$ can be used to differentiate between the coil-like and globule-like conformations of PNIPAM and PS in presence of water. Figure 2(a) shows the $\mathrm{R}_{\mathrm{g}}$ of an individual chain of 30-mer of PNIPAM (System A in Table 1) below and above its LCST. We note a difference in the temporal evolution of $\mathrm{R}_{\mathrm{g}}$ at the two temperatures. The mean value of $\mathrm{R}_{\mathrm{g}}$ of $\sim 15.6 \pm 0.4 \AA$ for PNIPAM 30 mer suggests a coil-like conformation of PNIPAM at $275 \mathrm{~K}$. On the other hand at $325 \mathrm{~K}$, the $\mathrm{R}_{\mathrm{g}}$ of $\sim 10.2 \pm 0.1 \AA$ represents a globule-like state of PNIPAM above its LCST. This observation is consistent with our previous studies conducted with PCFF force-field ${ }^{2}$ and other investigations 
performed by different research groups. ${ }^{42,55}$ For example, our results are consistent with an allatom MD study by Chiessi et al. where they reported a probability distribution of $\mathrm{R}_{\mathrm{g}}$ for 30 -mer of PNIPAM with a maximum of $12.5 \AA$ at $283 \mathrm{~K}$ (below the LCST) and $9.5 \AA$ at $323 \mathrm{~K}$ (above the LCST). ${ }^{42}$ In another computational study of 30-mer PNIPAM by Abbott et al., $\mathrm{R}_{\mathrm{g}}$ value of 10 $\AA$ at $330 \mathrm{~K}$ was reported. ${ }^{55}$

Figure 2 (b) shows that after 12 ns of the simulation, $R_{\mathrm{g}}$ of the 30 -mer PS fluctuates around the mean value (9.6 $\AA$ at $275 \mathrm{~K}$ and $11.0 \AA$ at $325 \mathrm{~K})$ at both temperatures, also listed in Table 2. This behavior is expected as the hydrophobic chain of PS is insoluble in water and leads to formation of a globule-like conformation. Note, pure PS chain is insoluble in water, however, our observations of globule-like state of PS are in excellent agreement with the recent computational and experimental findings on the chemically modified PS chains. ${ }^{48,56}$ Essafi et. al. found that the chain conformation evolves into the pearl shaped necklace shape in pure water, which makes it insoluble. Carrillo and Dobrynin ${ }^{48}$ reported that PS chains with 16 to 64 monomer units of PS collapse to form dense globule-like structures with $\mathrm{R}_{\mathrm{g}}$ values of 8-10 $\AA$ for a 32-mer of PS, consistent with our predictions here.

Table 2: Mean radius of gyration $\left(R_{g}\right)$ and its standard deviation shown for pure PNIPAM and the hybrid structures. For systems $A, C$, and D the $R_{g}$ values of only PNIPAM chains are listed. The mean $R_{g}$ is calculated from the values of $R_{g}$ recorded during the last 2 ns of the simulations.

\begin{tabular}{ccccc}
\hline System & \multicolumn{2}{c}{ PNIPAM (units) } & PS (units) & Average $(\AA)$ \\
\hline A & 275 K & & \\
B & 30 & 0 & $15.6 \pm 0.4$ \\
C & 0 & 30 & $11.0 \pm 0.4$ \\
D & 30 & 30 & $15.5 \pm 0.5$ \\
\hline A & 30 & 60 & $14.4 \pm 0.5$ \\
\hline B & & $35 \mathbf{~}$ & & $10.2 \pm 0.1$ \\
C & 30 & 0 & $9.6 \pm 0.1$ \\
D & 0 & 30 & $10.5 \pm 0.2$ \\
\hline
\end{tabular}

We now investigate the effect of number of PS chains on the coil-to-globule transition of PNIPAM in PNIPAM-co-PS co-polymers above its LCST. Here, we examine two block co- 
polymers of PNIPAM-co-PS: (i) a single chain of PS co-polymerized with a 30-mer of PNIPAM (system $\mathrm{C}$ in Table 1 and Figure 1) and (ii) two chains of PS co-polymerized with a 30-mer of PNIPAM (system D in Table 1 and Figure 1). Table 2 shows the mean values of $\mathrm{R}_{\mathrm{g}}$ for PNIPAM chains averaged over the last $2 \mathrm{~ns}$ of total simulation run. The mean $\mathrm{R}_{\mathrm{g}}$ at $275 \mathrm{~K}$ for PNIPAM chains in PNIPAM-co-PS co-polymers in systems C and D is in the range of $\sim 14.5-15.5 \AA$ that suggests a coil-like conformation of PNIPAM below its LCST. On the other hand, we find that the mean $\mathrm{R}_{\mathrm{g}}$ of the PNIPAM chain at $325 \mathrm{~K}$ in both the co-polymers $\mathrm{C}$ and $\mathrm{D}$ decreases to $\sim 8.5$ $10.2 \AA$. This reduction in the $\mathrm{R}_{\mathrm{g}}$ suggests that the PNIPAM undergoes a coil-to-globule transition with increasing temperature. Interestingly, we find that the system D with two PS chains exhibits the smallest mean $\mathrm{R}_{\mathrm{g}}$ value of $\sim 8.5 \AA$. We conjecture that the presence of the higher number of hydrophobic PS monomer in the PNIPAM-co-PS co-polymers results in the low $\mathrm{R}_{\mathrm{g}}$. The PS units disrupt the interaction between PNIPAM and water molecules, which leads to strong aggregation of hydrophobic groups of PNIPAM. We note that the $\mathrm{R}_{\mathrm{g}}$ of PS chains in PNIPAM-co-PS block co-polymers suggests globule-like conformation of PS 30 -mer both at $275 \mathrm{~K}$ and $325 \mathrm{~K}$.

We find that in all of the block co-polymers, PNIPAM exhibits a clear coil-to-globule transition above its LCST. This observation is in excellent agreement with the experimental studies of copolymers of PNIPAM where such coil-to-globule transitions are observed. ${ }^{33,34}$ Nuopponen et al. varied the mutual lengths of the blocks of PS and PNIPAM in the PNIPAM-co-PS co-polymers and their microcalorimetrical analysis suggested the dehydration and subsequent collapse of the PNIPAM chains in all samples. In another earlier report, reduction in the LCST of co-polymer chains is observed relative to pure PNIPAM chains. ${ }^{33}$

We present in Table 3 the end-to-end distance for 30-mer PNIPAM polymer chains for initial and final $2 \mathrm{~ns}$ of simulation at $275 \mathrm{~K}$ and $325 \mathrm{~K}$. The end-to-end distance of 30-mer PNIPAM does not show any significant differences between initial and final configurations below the LCST (at $275 \mathrm{~K}$ ). However, at $325 \mathrm{~K}$ the end-to-end of 30-mer for both pure chain of PNIPAM in system A and a chain of PNIPAM in PNIPAM-co-PS copolymer (in system C and D) reduces significantly. 
Table 3: End-to-end distance for pure PNIPAM and the co-polymer structures are listed. For systems $C$ and D, the values of only PNIPAM chains are listed. The mean end-to-end distance is calculated from the trajectories recorded during the first and the last $2 \mathrm{~ns}$ of the simulations.

\begin{tabular}{|c|c|c|c|c|}
\hline System & PNIPAM (units) & PS (units) & $\begin{array}{l}\text { End-to-end distance } \\
(\text { First 2ns) }(\AA)\end{array}$ & $\begin{array}{c}\text { End-to-end distance } \\
\text { (Last 2ns) }(\AA)\end{array}$ \\
\hline \multicolumn{5}{|c|}{$275 \mathrm{~K}$} \\
\hline $\bar{A}$ & 30 & 0 & 45.12 & 50.13 \\
\hline C & 30 & 30 & 46.23 & 48.62 \\
\hline D & 30 & 60 & 60.68 & 48.81 \\
\hline \multicolumn{5}{|c|}{$325 \mathrm{~K}$} \\
\hline $\mathrm{A}$ & 30 & 0 & 47.22 & 9.31 \\
\hline $\mathrm{C}$ & 30 & 30 & 44.52 & 27.63 \\
\hline D & 30 & 60 & 55.23 & 26.99 \\
\hline
\end{tabular}

(ii) Small Angle Neutron Scattering (SANS)

Small angle x-ray scattering (SAXS) and small angle neutron scattering (SANS) measurements have been widely used to study biomolecules and phase transitions in polymers. ${ }^{4-52}$ In a complex polymeric system such as PNIPAM-co-PS, both polymers exhibit a globule-like state above the LCST of PNIPAM. On the other hand, below its LCST, PNIPAM is in a coil-like state and PS is in a globule-like state. In such a scenario experimental SANS or SAXS spectra could be misleading in terms of predicting the coil-like state of PNIPAM below its LCST. In addition, experimentally observed spectra have traces of aggregates of polymer chains that might be consisting of different number of chains with various chain lengths. Thus, experimentally it is difficult to isolate a single polymer or a co-polymer chain in a solvent to calculate such a spectra, limiting our understanding of their structure and ability to design polymer networks with predefined shapes and geometries. We employ the CRYSON program to conduct the small angle neutron scattering (SANS) measurements from atomic trajectories of MD simulations to monitor the conformation of polymer chains below and above the LCST of PNIPAM. ${ }^{57}$

Figure 3 shows the SANS spectra for systems A, B, C, and D. In case of system A, with a 30mer PNIPAM only chain, the intensity for SANS spectra in the low $q$ region increases as temperature is raised from $275 \mathrm{~K}$ to $325 \mathrm{~K}$. This change can be attributed to the globule-like 
shape of the PNIPAM at $325 \mathrm{~K}$. Papagiannopoulos calculated the SANS curves for co-polymers of PNIPAM-co-PS from 0.5 and $0.7 \mathrm{mg} / \mathrm{ml}$ of PS-PNIPAM-PS in $\mathrm{D}_{2} \mathrm{O}$ at 4 different temperatures, namely, at $298 \mathrm{~K}, 303 \mathrm{~K}, 313 \mathrm{~K}$, and $323 \mathrm{~K}^{58}$ and observed similar increase in the intensity of low $q$ region as temperature is increased from $298 \mathrm{~K}$ to $323 \mathrm{~K}$, which they attributed to the temperature-induced clustering enhancement. They found that the aggregate radius is about 40-49 $\mathrm{nm}$ below the LCST and 32-34 $\mathrm{nm}$ above the LCST of PNIPAM. Additionally, similar increase in the peak intensities upon collapse of the structure above the LCST has been observed in the experimental SANS measurements for PNIPAM brush structures, hydrogels, and microgels. ${ }^{59-61}$ The shape of the SANS trace observed below the LCST of PNIPAM is consistent with those of rod-like macromolecules, suggesting that PNIPAM is in rod-like or coil-like state. $^{60,62}$ The $\mathrm{R}_{\mathrm{g}}$ calculated from the slope of the net intensities obtained with the CRYSON for PNIPAM 30-mer at $275 \mathrm{~K}$ and $325 \mathrm{~K}$ are $\sim 16.7 \AA$ and $\sim 9.9 \AA$, respectively. These values are similar to the values reported in Table 2. In the case of PS 30-mer, as shown in Figure 3 (b), no significant change is noted in the SANS measurements because PS chain is in globule form both at 275 and $325 \mathrm{~K}$. In the case of PNIPAM-co-PS and PS-PNIPAM-PS co-polymer systems for $275 \mathrm{~K}$, as shown in Figures 3 (c) and (d), a shoulder can be observed at $\sim 0.12$ and $\sim 0.15 \AA^{-1}$, respectively. This shoulder is attributed to the small clusters or coil-like state of PNIPAM chain. At $325 \mathrm{~K}$ this peak disappears suggesting that PNIPAM has undergone a coil-to-globule transition.

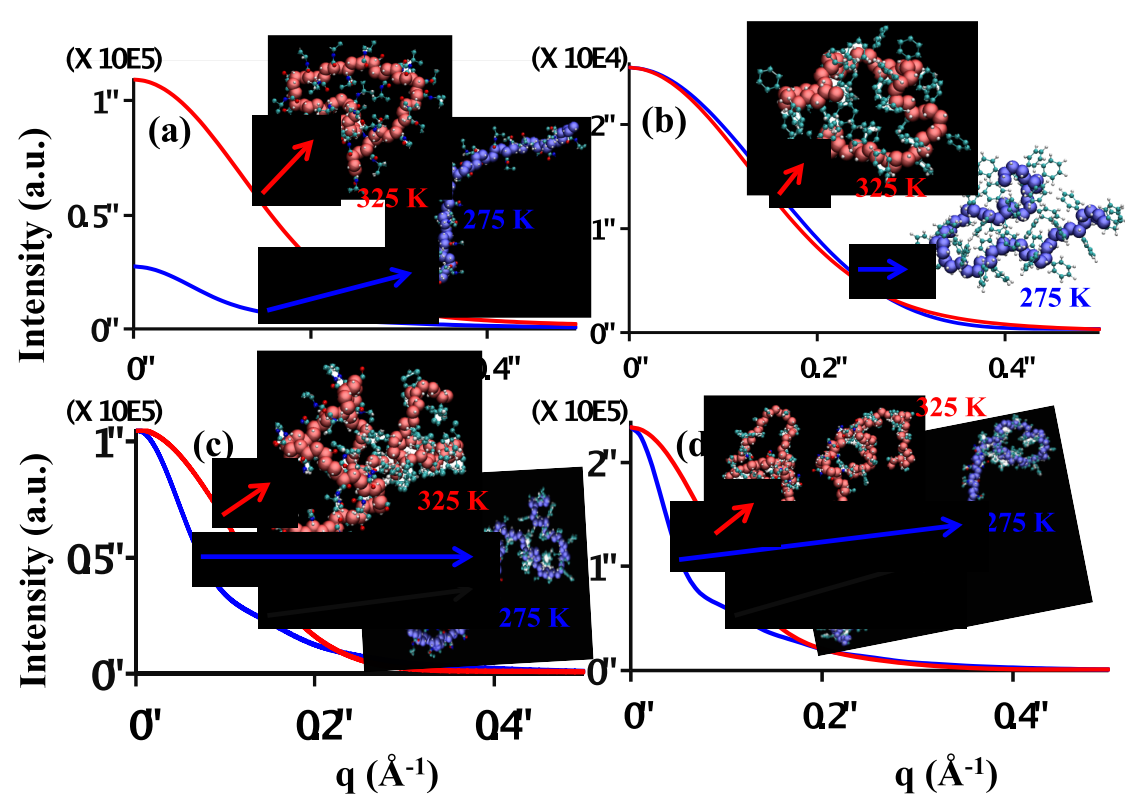


Figure 3: Results from small angle neutron scattering (SANS) calculated from the MD simulation trajectories at $275 \mathrm{~K}$ and $325 \mathrm{~K}$. (a), (b), (c), and (d) show the spectra for system A, $B, C$, and D, respectively described in Table 1. The corresponding polymer chain conformation is shown in inset. Backbones of polymer chains at $275 \mathrm{~K}$ and $325 \mathrm{~K}$ are shown in blue and red, respectively. The black arrow shows the coil-like region of PNIPAM in the spectra.

\section{(iii) Dynamic evolution of $R_{g}$ of PNIPAM as a function of the number of PS chains}

We further analyze the simulation trajectories of PNIPAM-co-PS co-polymer systems to study the dynamic evolution of $\mathrm{R}_{\mathrm{g}}$ of PNIPAM chains as a function of time and number of chains of PS. Figure 4 shows the variation in $\mathrm{R}_{\mathrm{g}}$ at $\sim 325 \mathrm{~K}$ for system $\mathrm{A}, \mathrm{C}$, and $\mathrm{D}$ with 0 , 30, and 60 monomer units (0-mer, 30-mer, and 60-mer) of a PS chain, respectively. It can be clearly seen that the time elapsed for PNIPAM chains to undergo a transition from a coil-like state to a globule-like state is different for every system. In particular, a transition time for 30-mer of PNIPAM to reach a globule-like state from a starting coil-like state decreases with increase in PS monomer units. PNIPAM chain with 0-mer, 30-mer, and 60-mer of PS chains exhibit transition from a coil-like state to a globule-like state in $\sim 30 \mathrm{~ns}, \sim 24 \mathrm{~ns}$, and $\sim 14 \mathrm{~ns}$, respectively. This observation suggests that presence of PS units facilitates the transition of PNIPAM from a coillike state to a globule-like state. This could be because PS units might be disrupting the interactions between PNIPAM and water molecules. Experimentally, it is well known that increase in concentration of hydrophobic moieties beyond a critical concentration in PNIPAM co-polymers can result in water insoluble polymers. ${ }^{36-38}$ Similarly, in the present study we find that with increase in number of PS units a coil-to-globule transition time for PNIPAM decreases.

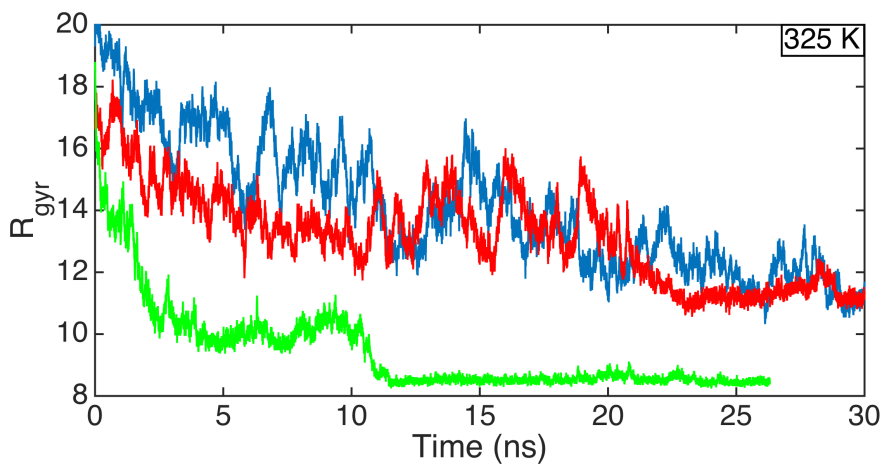


Figure 4: Dynamic evolution of $R_{g}$ of PNIPAM at $325 \mathrm{~K}$ in PNIPAM-co-PS co-polymer chains with 0 (System A), 30 (System C), and 60 (System D) PS units are shown in blue, red, and green respectively.

\section{(iv) Distribution of PS monomer units near PNIPAM chain}

The time evolution of $\mathrm{R}_{\mathrm{g}}$ shown in Figure 4 suggests that system D (with 2 chains of PS) undergoes a coil-to-globule transition before system C (with 1 PS chain) and system A (with 0 PS chain). We further elaborate the role played by PS monomers in accelerating this coil-toglobule transition by calculating the average distribution of PS units near the central monomer of PNIPAM (see schematic in Figure 1 for the location of the central PNIPAM monomer) after $\sim 20$ ns simulation run. The trajectories are averaged during the last $1 \mathrm{~ns}$ (1000 frames) of the simulation. The distribution of PS monomers around the central monomer of PNIPAM for both system C and system D are shown in Figure 5.

We see from Figure 5 (a) and (b) that for system C, the average distance between PS monomers and the central PNIPAM monomers is $\sim 35 \AA$ and $\sim 20 \AA$ at $275 \mathrm{~K}$ and $325 \mathrm{~K}$, respectively. Likewise, for system $\mathrm{D}$ at $275 \mathrm{~K}$, as shown in Figure 5 (c), the graph shows a broad distribution of PS monomers ranging from the distance of $\sim 10 \AA$ to $\sim 40 \AA$. In contrast, at $325 \mathrm{~K}$ a narrow distribution of PS monomers with an average value of $\sim 15 \AA$ can be seen from Figure 5 (d). In both systems $\mathrm{C}$ and $\mathrm{D}$ at $325 \mathrm{~K}$ the presence of increased number of PS monomers within a distance of $\sim 20 \AA$ disrupts the structure of water near PNIPAM monomers, significantly altering the structure of proximal water near PNIPAM and changing the interactions between PNIPAM and proximal water molecules. 

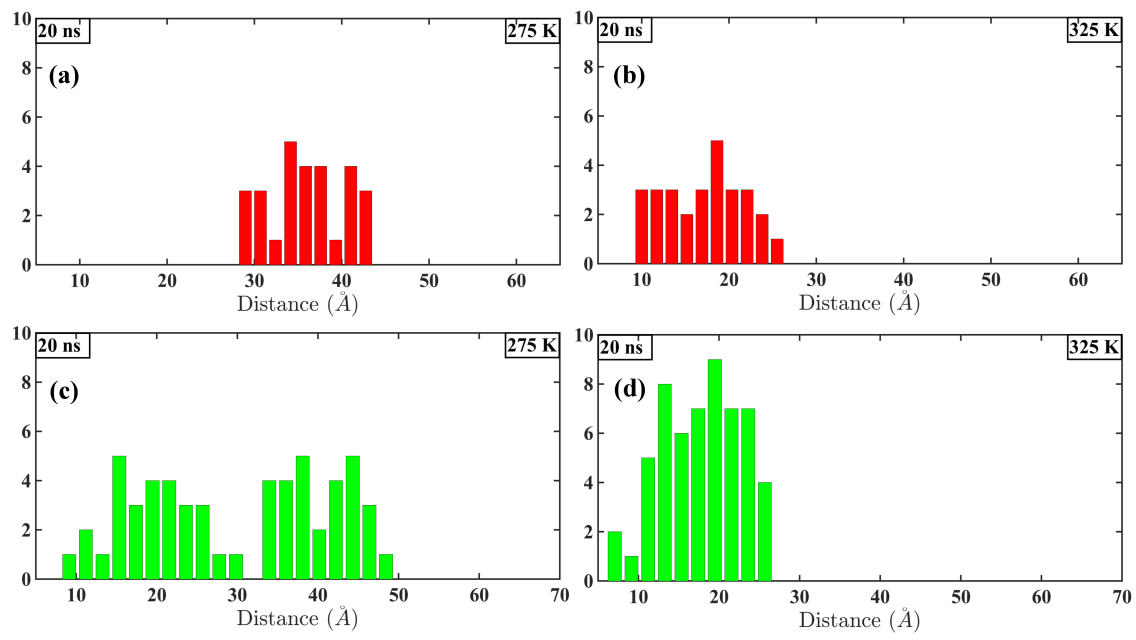

Figure 5: Distribution of PS monomers around the central monomer of PNIPAM chain after 20 ns for system C (a) at $275 \mathrm{~K}$ and (b) at $325 \mathrm{~K}$ and for system D at (c) at $275 \mathrm{~K}$ and (d) at $325 \mathrm{~K}$. PNIPAM-co-PS co-polymer chains with 30 (System C), and 60 (System D) PS monomers are shown in red, and green respectively.

\section{B. Structure and Dynamics of Water}

\section{(i) Radial Distribution Function (RDF) of Water and Co-polymer}

We calculate the RDF of water molecules with respect to the atoms in the backbone and side chain of PNIPAM to analyze the structure of water near the PNIPAM chain. Figure 6 (a) depicts the RDF of oxygen of water molecules (ow) with the carbon on the backbone (ch $2 b)$ of PNIPAM 30-mer system A. The RDF of oxygen atom (op) of carbonyl group of PNIPAM in system A and water oxygen (ow) is shown in Figure 6 (b). The RDF analysis is performed using atomic trajectories during the last $2 \mathrm{~ns}$ of the simulation. In the case of RDF for ch $2 b$ with op a welldefined peak for first neighbors is located at $\sim 5.00 \AA$ both at $275 \mathrm{~K}$ and $325 \mathrm{~K}$ (Figure 6 (a)). In addition to the first peak, we also observe a shoulder at $\sim 9 \AA$ at $275 \mathrm{~K}$ that is absent at $325 \mathrm{~K}$. $\mathrm{RDF}$ indicates short-range structure through positional correlation between polymer and water, which decreases with an increase in the temperature from $275 \mathrm{~K}$ to $325 \mathrm{~K}$. The dramatic decrease in the peak height of the first peak and absence of the shoulder at $325 \mathrm{~K}$ suggests that the structure of water near polymer is significantly different at the two temperatures. This prediction indicates that at $325 \mathrm{~K}$ water is expelled from the globule-like conformation of PNIPAM (see Figure 1 (a)). In the case of op-ow RDF, both at $275 \mathrm{~K}$ and $325 \mathrm{~K}$ three peaks for first, second, 
and third neighbors are recorded at $\sim 3 \AA, \sim 5 \AA$, and $\sim 7 \AA$, respectively. In addition, a small shoulder is observed at $\sim 4.2 \AA$ only at $275 \mathrm{~K}$. Similar to the ch $2 b$-ow RDF, we find that the peak height for all three peaks decreases at $325 \mathrm{~K}$ as compared to $275 \mathrm{~K}$. This observation suggests different structural arrangement of water near backbone and side chain of PNIPAM below and above the LCST and strongly supports the globule-like conformation of PNIPAM at $325 \mathrm{~K}$.
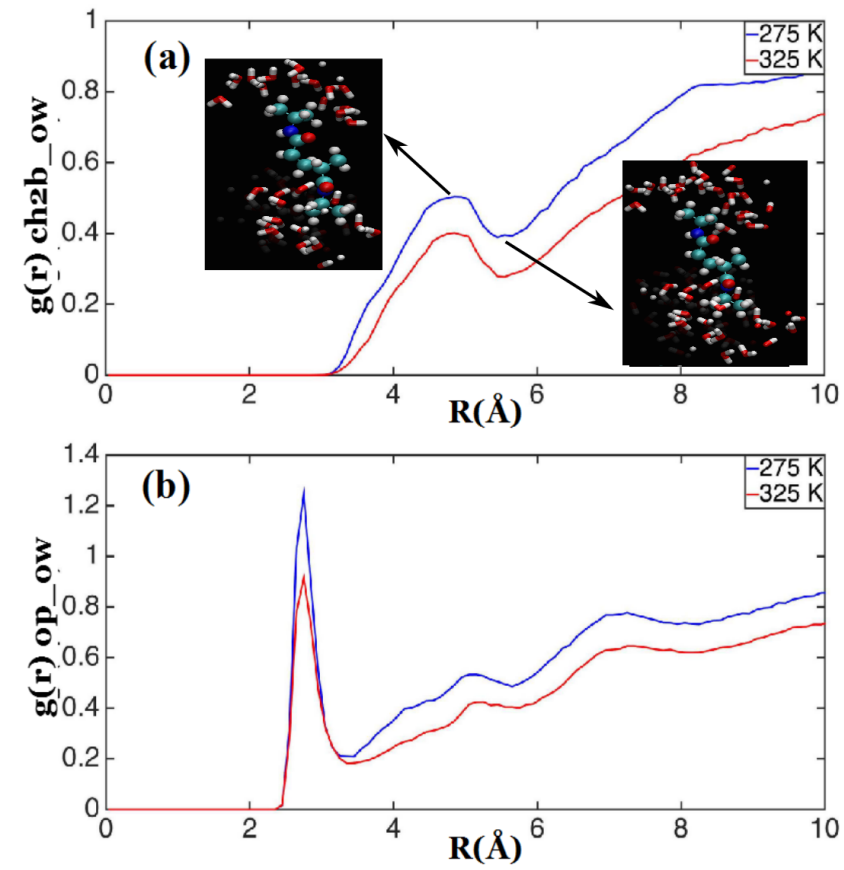

Figure 6: Radial distribution function (RDF) of (a) the carbon atoms in the backbone (ch2b) of PNIPAM chain in system A to water oxygen (ow) and (b) oxygen atom (op) of carbonyl group of PNIPAM in system A and water oxygen (ow) at $275 \mathrm{~K}$ and $325 \mathrm{~K}$.

Figure 7 shows the RDF for carbon in the backbone (ch2b) of PS 30-mer from system B and oxygen of water (ow) both at $275 \mathrm{~K}$ and $325 \mathrm{~K}$. The ch2b-ow RDF shows a broad shoulder at $\sim 4-$ $5 \AA$ both at $275 \mathrm{~K}$ and $325 \mathrm{~K}$, while the height of the peak decreases moderately with increase in temperature. Comparing the RDFs of carbon in backbone of PNIPAM (Figure 6 (a)) and PS (Figure 7) with oxygen of water suggests that PNIPAM is more hydrated as compared to PS, since PNIPAM is relatively more hydrophilic in nature and additional water molecules are present in its neighborhood both at $275 \mathrm{~K}$ and $325 \mathrm{~K}$. In other words, the hydrophobic nature of PS repels water molecules and dehydrates the polymer chain. 


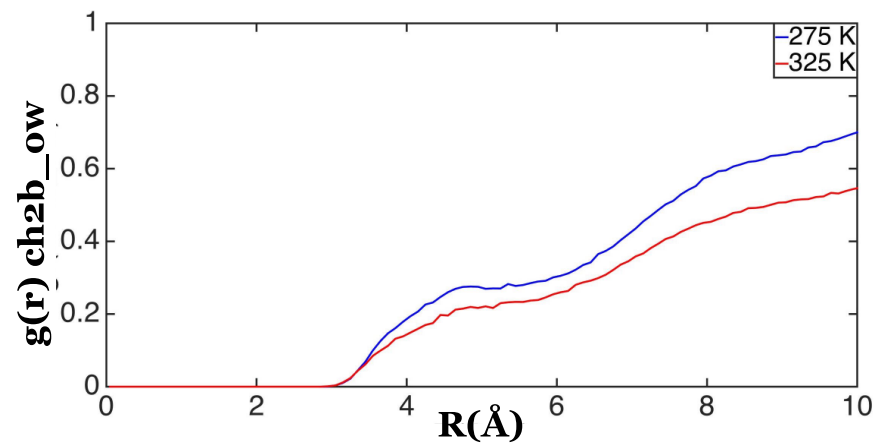

Figure 7: Radial distribution function (RDF) of the backbone (ch2b) of polystyrene (PS) polymer chain to water oxygen (ow) at $275 \mathrm{~K}$ and $325 \mathrm{~K}$.
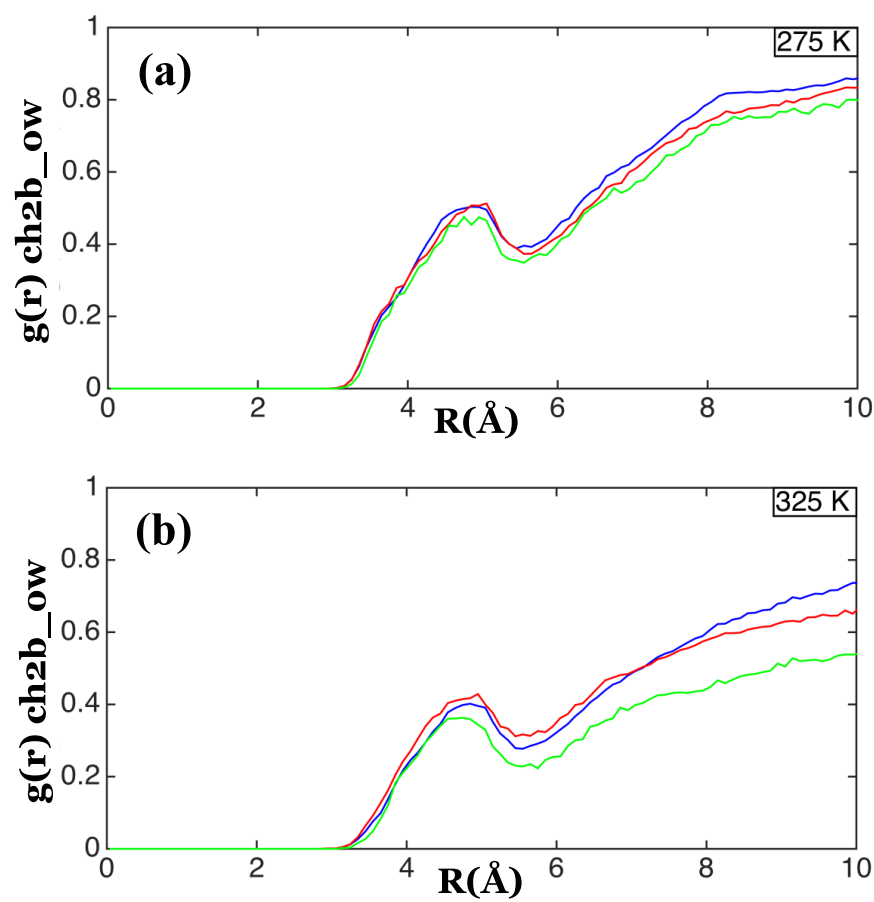

Figure 8: Radial distribution function (RDF) of the backbone (ch2b) with the oxygen (ow) of water (a) at $275 \mathrm{~K}$ and (b) at $325 \mathrm{~K}$. Blue, red, and green curves represent the RDFs for systems $A, C$ and $D$, respectively.

Figures 8 (a) and (b) show the RDFs at $275 \mathrm{~K}$ and $325 \mathrm{~K}$ for carbon in the backbone (ch2b) of PNIPAM with oxygen (ow) of water molecules for the PNIPAM-co-PS co-polymers with 
different number of PS monomers. A broad peak is observed at $\sim 4-5 \AA$ for both $275 \mathrm{~K}$ and 325 K. The shoulder at $\sim 9 \AA$ in the $c h 2 b$-ow RDF for PNIPAM in system A at $275 \mathrm{~K}$ ceases to exist with increase in the number of PS monomers. The PNIPAM chain dehydrates with increase in number of PS units. We also find that with increase in the temperature the height of the peak observed at $\sim 4-5 \AA$ decreases significantly and the shoulder at $\sim 9 \AA$ is non-existent implying that the structure of water near the backbone of PNIPAM is different at $325 \mathrm{~K}$ as compared to $275 \mathrm{~K}$ and potentially driven by the coil-to-globule transition of PNIPAM.
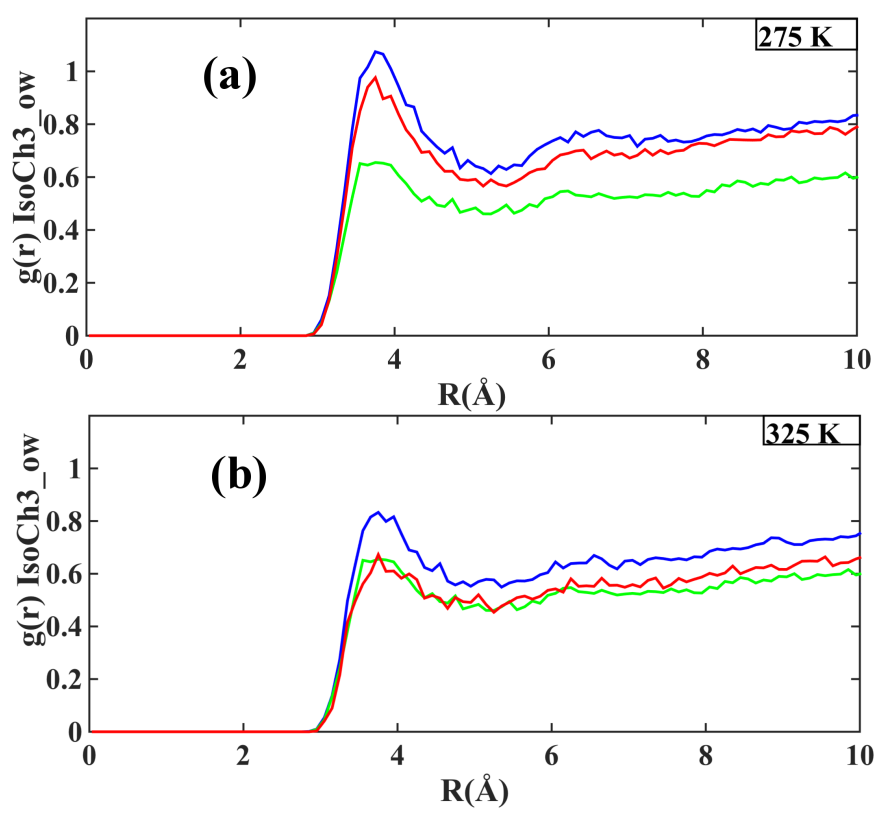

Figure 9: Radial distribution function (RDF) of the isopropyl carbon (IsoCh3) with the oxygen (ow) of water (a) at $275 \mathrm{~K}$ and (b) at $325 \mathrm{~K}$. Blue, red, and green curves represent the RDF for systems $A, C$ and $D$, respectively.

Figures 9 (a) and (b) show the RDF at $275 \mathrm{~K}$ and $325 \mathrm{~K}$ for end carbon of the isopropyl group (IsoCh3) of PNIPAM with oxygen (ow) of water molecules, respectively, for the PNIPAM-coPS co-polymers with different number of PS monomers. The RDF peak height decreases at 325 $\mathrm{K}$ for all the systems relative to $275 \mathrm{~K}$. The first peak observed at $\sim 3.8 \AA$ decreases with increase in temperature and with the number of PS units, similar ch2b-ow RDF. Similar observations for second peaks are also noted from Figures 10 (a) and (b), which show the RDF for Nitrogen atom $(N)$ of PNIPAM with oxygen (ow) of water molecules for the PNIPAM-co-PS co-polymers with 
different number of PS monomers. We conclude from the results of all the RDFs that PNIPAM chain undergoes enhanced dehydration with increase in temperature.
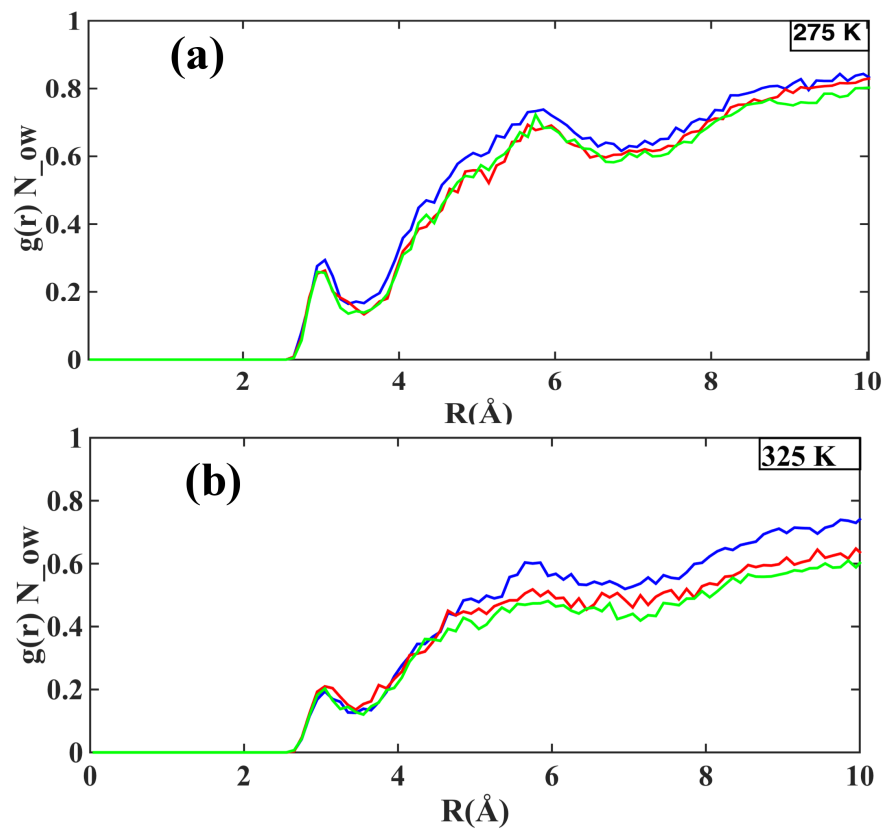

Figure 10: Radial distribution function (RDF) of the nitrogen atom (N) of PNIPAM with the oxygen (ow) of water (a) at $275 \mathrm{~K}$ and (b) at $325 \mathrm{~K}$. Blue, red, and green curves show the RDFs for systems $A, C$ and $D$, respectively.

\section{(ii) Degree of Hydration of Co-polymers}

We now evaluate the extent of hydration by analyzing the simulation trajectories for water molecules in the first hydration shell of PNIPAM and PS polymer chains. Note, the first hydration shell for each polymer chain is decided based on the location of the first minimum in the RDF for polymer atoms and oxygen of water. For example, first hydration shell of the backbone of PNIPAM is decided, to be $<=\sim 5.4 \AA$, based on the location of first minimum in the RDF for carbon atom of backbone in PNIPAM and oxygen atom of water (ow). Figure 11 (a) and (b) show the snapshots of water molecules in the first hydration shell of PNIPAM in system A, at the end of simulation run, at $275 \mathrm{~K}$ and $325 \mathrm{~K}$. As illustrated in Figure 11 (a) the PNIPAM chain is well hydrated below the LCST. The presence of water and its atomic level structural arrangement in the first hydration shell of PNIPAM monomers at $275 \mathrm{~K}$ is evident from the 
magnified snapshot shown as an inset in Figure 11 (a). We find that water molecules near the hydrophilic groups are arranged in such a manner that they facilitate the hydrogen bond between water and PNIPAM monomers. On the other hand, water favors the formation of a cage-like structure around a hydrophobic group due to the latter's inability to form hydrogen bonds. This structural arrangement of water between two monomers promotes the coil-like structure of PNIPAM below its LCST. In contrast, at $325 \mathrm{~K}$, as presented in Figure 11 (b) the ordered framework of water collapses due to the globule-like state of PNIPAM chain above its LCSTK. Figures 11 (c) and (d) show the representative illustrations of water molecules in the first hydration shell of PS in system B, at the end of simulation run of 20 ns at $275 \mathrm{~K}$ and $325 \mathrm{~K}$, respectively. While PS chain is surrounded by water molecules the hydrophobic nature of PS monomers expels water from the core of the globule-like structure at both temperatures.
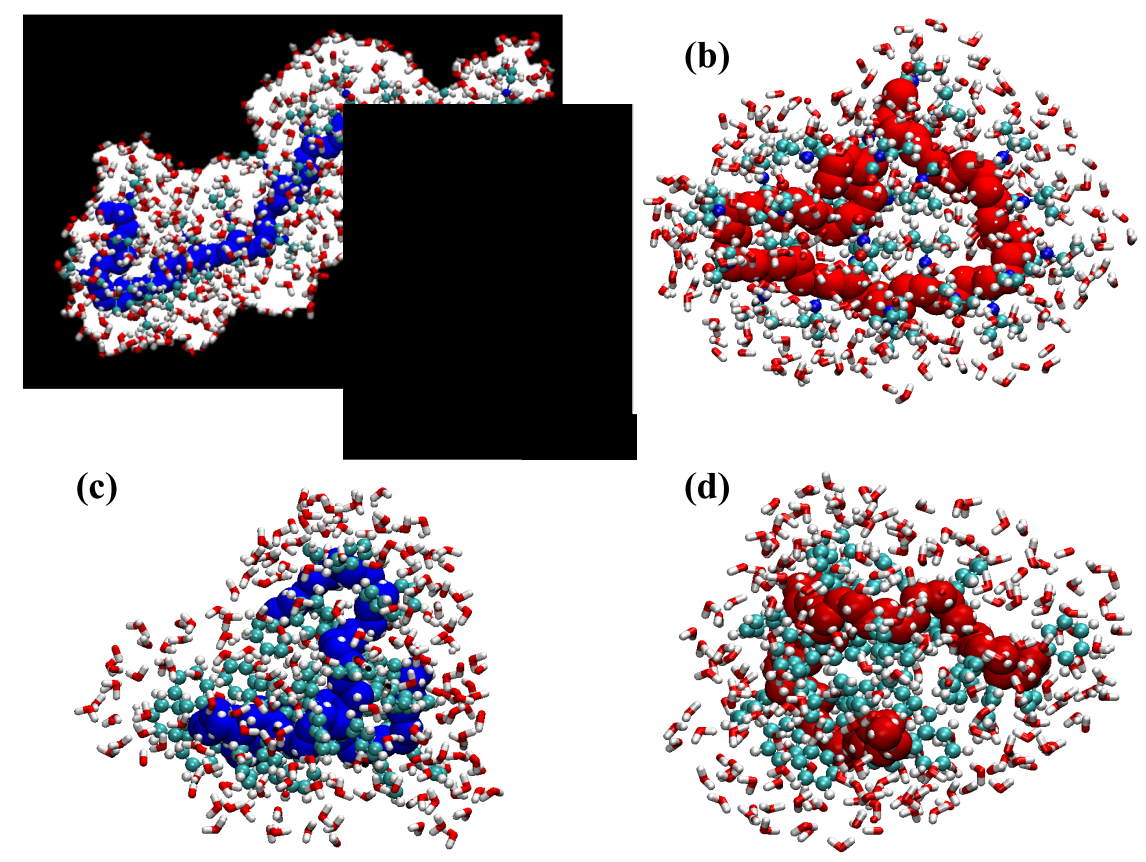

Figure 11: First hydration shell of PNIPAM (a) at $275 \mathrm{~K}$ and (b) at $325 \mathrm{~K}$ and PS (c) at $275 \mathrm{~K}$ and (d) at $325 \mathrm{~K}$. The snapshot in (a) shows that in a coil-like state PNIPAM is hydrated while in the globule-like state in (b) and for PS in (c) and (d), there is absence of water in the core of the polymer globule. Figure (a) also shows a magnified inset of a PNIPAM monomer at $275 \mathrm{~K}$ in presence of first hydration shell of water describing a cage-like structure of water around the isopropyl group of PNIPAM monomer. 
We further quantify the change in the extent of hydration of polymer chains by calculating the number of water molecules in the first hydration shell of PNIPAM chains. Table 4 summarizes the average number of water molecules present in the first hydration shell for the systems investigated. In both pure PNIPAM chain (system A) and PNIPAM chain in PNIPAM-co-PS copolymers (systems $\mathrm{C}$ and D) the average number of water molecules near both the isopropyl group and backbone of PNIPAM decrease with increase in temperature. Below the LCST of PNIPAM, we find that the isopropyl group has $\sim 11.8$ water molecules per monomer in all the systems, which is in excellent agreement with the dielectric spectroscopy experimental value of 11 water molecules. ${ }^{2,63}$ On the other hand, above the LCST, the number of water molecules in the first hydration shell of side chain reduces to $\sim 9$ corroborating that the structural arrangement of water molecules near hydrophobic groups present in the side chain of PNIPAM is significantly different below and above the LCST. The number of water molecules obtained in our study $(\sim 11.8 \pm 0.4$ and $\sim 9.0 \pm 0.3$ at $325 \mathrm{~K}$ and $275 \mathrm{~K}$, respectively) are consistent with the values reported by Chiessi et al. for 30-mer PNIPAM with different tacticities. ${ }^{42}$ They found that a monomer unit of PNIPAM with 45\% meso-diad tacticity has $\sim 12.7$ and $\sim 10.4$ water molecules at $283 \mathrm{~K}$ and $323 \mathrm{~K}$.

Table 4: Number of water molecules in the first hydration shell at $275 \mathrm{~K}$ and $325 \mathrm{~K}$ (Data are averaged over last 5 ns of the production run).

\begin{tabular}{|c|c|c|c|c|c|}
\hline $\begin{array}{c}\text { No. of } \\
\text { PNIPAM } \\
\text { monomers }\end{array}$ & $\begin{array}{l}\text { No. of PS } \\
\text { monomers }\end{array}$ & System & $\begin{array}{c}\text { Temperature } \\
\text { (K) }\end{array}$ & $\begin{array}{l}\text { No. of water near } \\
\text { isopropyl group of } \\
\text { PNIPAM side chain }\end{array}$ & $\begin{array}{c}\text { No. of water } \\
\text { near backbone }\end{array}$ \\
\hline \multirow[t]{2}{*}{30} & 0 & A & 275 & $11.8 \pm 0.4$ & $9.7 \pm 0.3$ \\
\hline & & & 325 & $9.0 \pm 0.3$ & $7.0 \pm 0.7$ \\
\hline \multirow[t]{2}{*}{0} & 30 & B & 275 & NA & $8.9 \pm 0.6$ \\
\hline & & & 325 & NA & $7.2 \pm 0.4$ \\
\hline \multirow[t]{2}{*}{30} & 30 & $\mathrm{C}$ & 275 & $11.4 \pm 0.2$ & $8.9 \pm 0.3$ \\
\hline & & & 325 & $8.9 \pm 0.2$ & $6.6 \pm 0.4$ \\
\hline \multirow[t]{2}{*}{30} & 60 & $\mathrm{D}$ & 275 & $11.5 \pm 0.2$ & $8.4 \pm 0.2$ \\
\hline & & & 325 & $8.8 \pm 0.2$ & $5.7 \pm 0.3$ \\
\hline
\end{tabular}


Additionally, as listed in Table 4, for systems A, C, and D, the numbers of water molecules near the backbone of PNIPAM chains reduce at $325 \mathrm{~K}$ as compared to $275 \mathrm{~K}$. Specifically, with increase in temperature the number of water molecules reduce from $\sim 9.7$ to $\sim 7.0, \sim 8.9$ to $\sim 6.6$, and $\sim 8.4$ to $\sim 5.7$ for systems $\mathrm{A}, \mathrm{C}$, and $\mathrm{D}$ respectively. This prediction further asserts that with increase in both temperature and number of PS units in PNIPAM-co-PS co-polymers the extent of dehydration of the backbone of PNIPAM enhances. For the 30-mer PS chain (system B), 8.9 and $\sim 7.2$ water molecules are present per monomer at $275 \mathrm{~K}$ and $325 \mathrm{~K}$. Similar number of water molecules are observed around the PS chains for systems C and D.

Next, we analyze the simulation trajectories to examine the dynamic evolution of the hydration shell of PNIPAM as a function of number of PS units. Figure 12 shows the variation for number of water molecules per monomer of PNIPAM during a production run at $325 \mathrm{~K}$. In the beginning of the simulation $\sim 13$ number of water molecules are present in the first hydration shell of PNIPAM in all three systems, A, C, and D. However, with time, system D with 60-mer of PS, dehydrates after $\sim 14 \mathrm{~ns}$ (number of water molecules reduce to $\sim 9.0$ ). The systems with 30-mer and 0 -mer of PS, namely systems $\mathrm{C}$ and A, dehydrate after $\sim 24$ and $\sim 34$ ns. These time windows coincide with the time windows where the conformational transition in the PNIPAM chain is observed (shown previously in Figure 4). Such a finding suggests that a coil-to-globule transition in PNIPAM is correlated with the change in structure of proximal water. The increase in number of PS monomers accelerates the dehydration of PNIPAM chain, which we conjecture is due to the changes in the interaction between PNIPAM and proximal water driven by the hydrophobic nature of PS monomers.

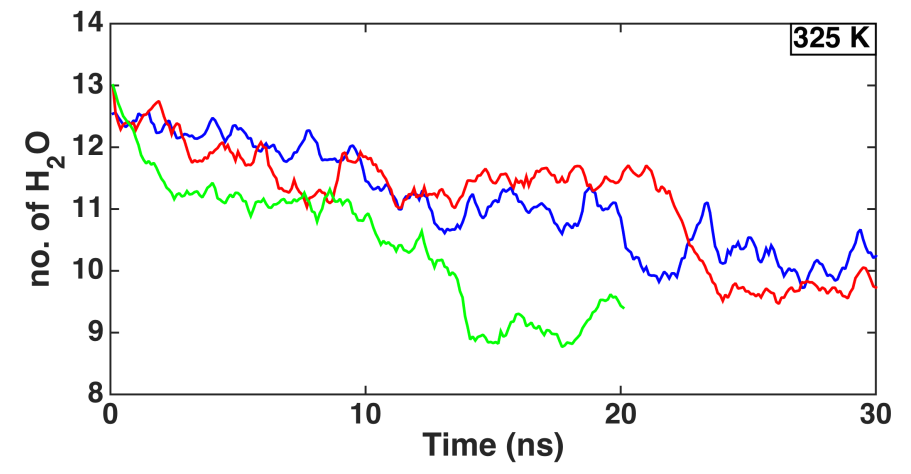


Figure 12: Number of water molecules in first hydration shell near the hydrophobic groups at both 275 and $325 \mathrm{~K}$. Blue, red, and green curves represent the RDFs for systems A, C and D, respectively.

\section{(iii) Residence time Probability $\left(\boldsymbol{P}_{\text {res }}(t)\right)$ of Water}

Figure 13 shows the residence probability $\left(\mathrm{P}_{\text {res }}\right)$ for water molecules near the hydrophilic groups of PNIPAM in systems A, C, and D both below and above the LCST. The $\mathrm{P}_{\text {res }}$ is calculated by the continuous definition and is the measure of how long one water molecule, on average, resides within a first hydration shell formed around the polymer. As seen in Figure 13, the residence time strongly depends on temperature and presence of PS chains. $P_{\text {res }}(t)$ decreases in presence of PS chains insinuating that PNIPAM-water interaction weakens in presence of PS chains. Figure 13 also suggests that water molecules in the first hydration shell (within $5.4 \AA$ of $\mathrm{C}=\mathrm{O}$ and $\mathrm{N}-\mathrm{H}$ groups in the polymer side chain) reside near the hydrophilic groups significantly longer for PNIPAM present in system C (PNIPAM chain at the end of a PS chain) relative to system D (PNIPAM chain in between 2 PS chains), both below and above the LCST. We conjecture that the PNIPAM chain in system D has weaker interactions with proximal water molecules due to presence of two hydrophobic chains of PS, resulting in faster dehydration of PNIPAM chains. Comparing Figures 13 (a) and (b) we find that the residence time for all the simulated polymer systems above the LCST is lower than that for simulations below LCST. In other words, the $\mathrm{P}_{\text {res }}(\mathrm{t})$ decays much slower at $275 \mathrm{~K}$ than at $325 \mathrm{~K}$. The slow decay of $\mathrm{P}_{\text {res }}(\mathrm{t})$ below LCST indicates energetically stable conformations of water molecules in the first hydration shell of polymer with pronounced water-polymer correlation. This hydrophilic nature of PNIPAM chains below the LCST assists PNIPAM to form stronger hydrogen bonds with water, resulting in slower decay of water molecules. This stronger hydrogen bonding enables water molecules to bind with PNIPAM for longer time.
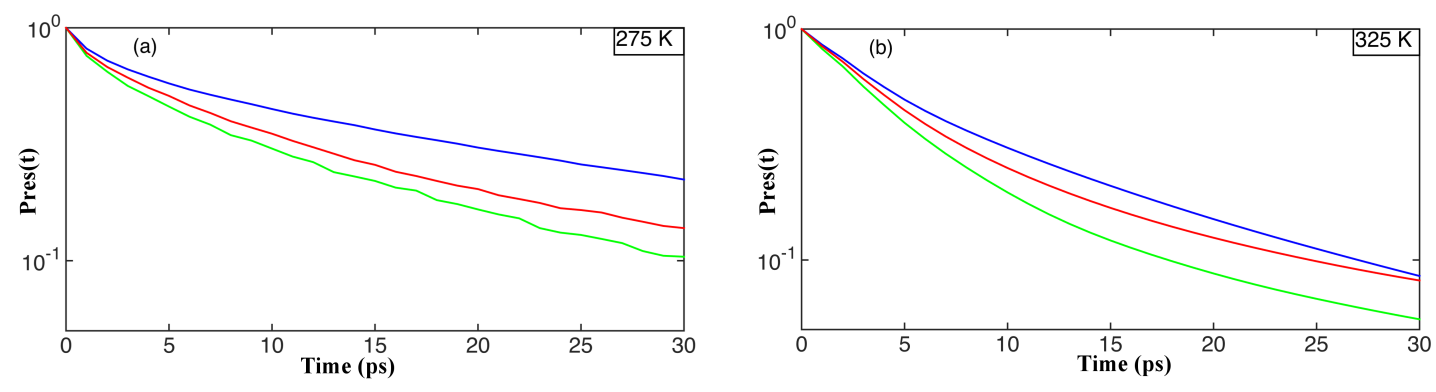

$-25-$ 
Figure 13: Semi-logarithmic distribution of residence probability of water near the hydrophilic region (a) at $275 \mathrm{~K}$ and (b) $325 \mathrm{~K}$. Blue, red, and green curves represent the RDFs for systems $A, C$ and $D$, respectively. System description is listed in Table 1.

\section{(iv) Hydrogen Bond Analysis}

Hydrogen bonding analysis is performed by using the geometric criteria as described in methods section above. Here, since only PNIPAM is capable of forming hydrogen bonds with water, the hydrogen bonds are classified into three types based on donor-acceptor pair: (1) polymer $\left(\left(\mathrm{N}_{\mathrm{pol}}{ }^{-}\right.\right.$ ) $\left.\mathrm{H}_{\mathrm{pol}}\right)$ - polymer $\left(\mathrm{O}_{\mathrm{pol}}\left(=\mathrm{C}_{\mathrm{pol}}\right)\right)$, (2) polymer $\left(\left(\mathrm{N}_{\mathrm{pol}}-\right) \mathrm{H}_{\mathrm{pol}}\right)$ - water $\left(\mathrm{O}_{\mathrm{W}}\right)$, and $(3)$ water $\left(\left(\mathrm{O}_{\mathrm{W}}-\right) \mathrm{H}_{\mathrm{W}}\right)$ - polymer $\left(\mathrm{O}_{\mathrm{pol}}\left(=\mathrm{C}_{\mathrm{pol}}\right)\right)$. The results of these analyses both below and above the LCST for systems $\mathrm{A}, \mathrm{C}$, and $\mathrm{D}$, by the definition of intermittent autocorrelation function, are shown in Figures 14 (a)-(f).

In case of type (1) hydrogen bonds, the polymer is both the donor and acceptor. As shown in Figures 14 (a)-(f), for the systems A, C, and D, the hydrogen bonds for type (1) decays faster at $275 \mathrm{~K}$ as compared to $325 \mathrm{~K}$. The slower decay at $325 \mathrm{~K}$ is attributed to the globule-like conformation that leads to strong hydrogen bonding in polymer. Comparison of types (2) and (3) hydrogen bonds, where polymer is either donor or acceptor respectively, reveals that the decay rate of hydrogen correlation for type (3) is faster as compared to that for type (2) in systems A and $\mathrm{C}$. This finding suggests that the hydrogen bond formed between hydrogen of amide group $\left(\mathrm{N}_{\mathrm{pol}}\left(-\mathrm{H}_{\mathrm{pol}}\right)\right)$ and water oxygen $\left(\mathrm{O}_{\mathrm{W}}\right)$ is more stable than that between carbonyl oxygen $\left(\mathrm{O}_{\mathrm{pol}}\right)$ and hydrogen of water $\left(\mathrm{H}_{\mathrm{W}}\right)$. We find that the decay rates of hydrogen bond correlation is slower for pure PNIPAM as compared to PNIPAM-co-PS co-polymers. Interestingly, the hydrogen bond decays faster in presence of PS chains in the co-polymers implying that water-polymer interactions becomes weaker with increase in number of PS chains in PNIPAM-co-PS copolymers. In other words, with an increase in the PS chains, water forms less stable hydrogen bond network with PNIPAM and with itself. We also note that for system A (PNIPAM chain only) the types (2) and (3) hydrogen bond correlations, cross each other both below and above the LCST. However, with increase in number of PS chains types (2) and (3) hydrogen bond correlations do not cross each other for majority of the simulation time suggesting that the presence of PS chains does influence the hydrogen bond interactions between water and polymer 
both below and above the LCST. The variation of hydrogen bond autocorrelation function calculated for type (1), (2) and (3) shows similar characteristics with earlier studies. ${ }^{54}$
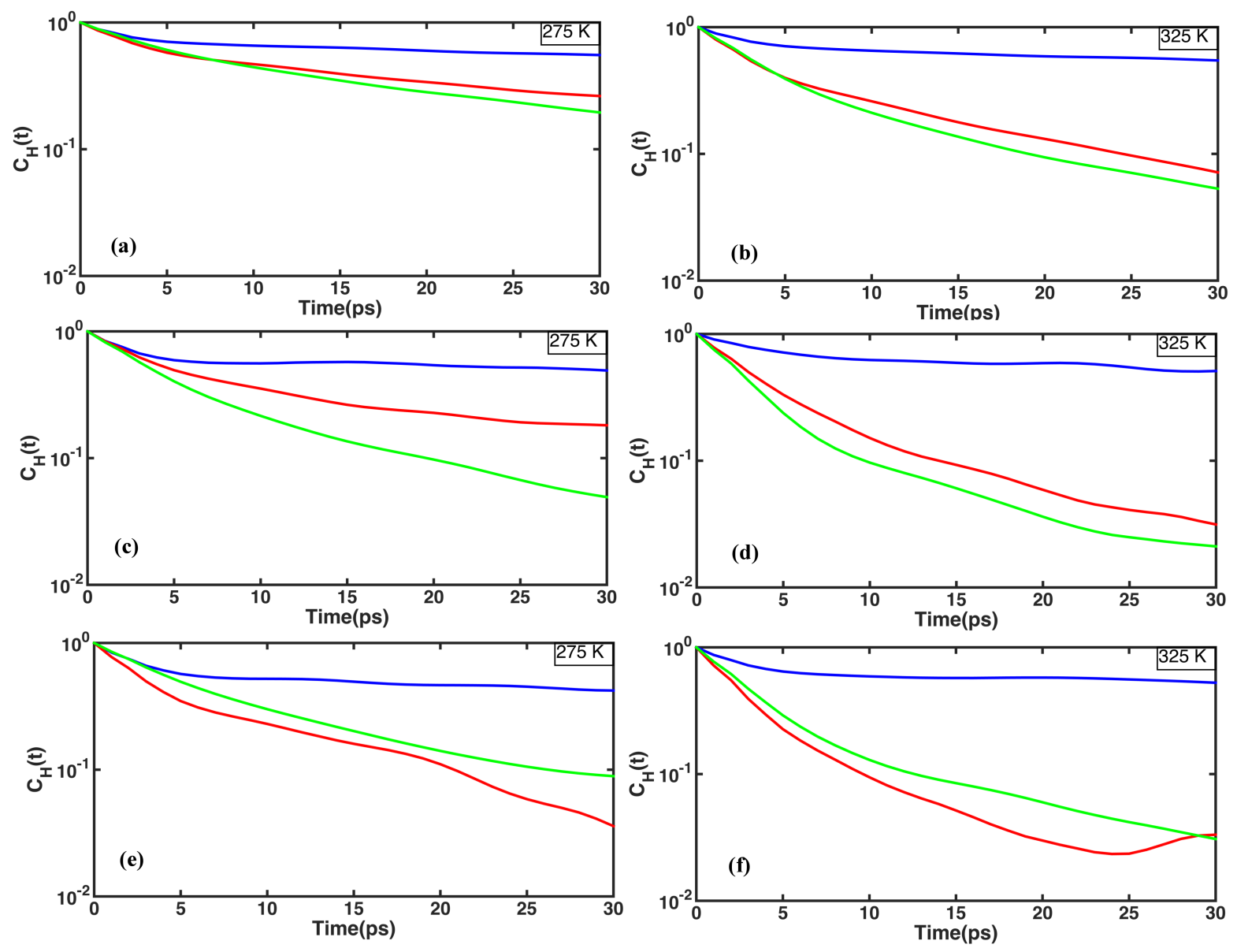

Figure 14: Semi-logarithmic hydrogen bond life-time autocorrelation function for polymerpolymer (type (1), blue), polymer-water (type (2), red) and water-polymer (type (3), green) combinations of donor-acceptor pairs, at $275 \mathrm{~K}$ for (a) system A, (c) system C, and (e) system D and at $325 \mathrm{~K}$ for (b) system $A$, (d) system $C$, and (f) system D, respectively.

Table 5: The number of hydrogen bonds for pure PNIPAM and the co-polymer structures are listed. For systems $A, C$, and $D$ the number of hydrogen bonds are reported for three cases - (1) polymer $((\mathrm{Npol}-) \mathrm{Hpol})$ - polymer $(\mathrm{Opol}(=\mathrm{Cpol}))$, (2) polymer $((\mathrm{Npol}-\mathrm{Hpol})-$ water $(\mathrm{OW})$, and (3) water $((\mathrm{OW}-) \mathrm{HW})$ - polymer $(\mathrm{Opol}(=\mathrm{Cpol}))$. The values are calculated during the last $2 \mathrm{~ns}$ of the simulations. 


\begin{tabular}{|c|c|c|c|c|c|}
\hline System & $\begin{array}{c}\text { PNIPAM } \\
\text { (units) }\end{array}$ & PS (units) & $\begin{array}{c}\text { H-bonds } \\
\text { (Pol - Pol) }\end{array}$ & $\begin{array}{c}\text { H-bonds } \\
\text { (Pol - wat) }\end{array}$ & $\begin{array}{c}\text { H-bonds } \\
\text { (wat - Pol) }\end{array}$ \\
\hline \multicolumn{6}{|c|}{$275 \mathrm{~K}$} \\
\hline $\mathrm{A}$ & 30 & 0 & 0. & 11 & 13 \\
\hline $\mathrm{C}$ & 30 & 30 & 3 & 9 & 12 \\
\hline $\mathrm{D}$ & 30 & 60 & 3 & 9 & 12 \\
\hline \multicolumn{6}{|c|}{$325 \mathrm{~K}$} \\
\hline $\mathrm{A}$ & 30 & 0 & 2 & 10 & 11 \\
\hline $\mathrm{C}$ & 30 & 30 & 2 & 6 & 10 \\
\hline $\mathrm{D}$ & 30 & 60 & 2 & 7 & 9 \\
\hline
\end{tabular}

Finally, we report in Table 5 the number of hydrogen bonds formed during the last $2 \mathrm{~ns}$ of the simulation. As expected the number decreases at $325 \mathrm{~K}$ for all systems and the PNIPAM unit becomes hydrophobic in all of them. Furthermore, we find less number of hydrogen bonds formed in the bigger systems. These predictions compare well with the number of hydrogen bonds obtained from all-atom MD simulations in the literature. ${ }^{64}$.

\section{IV) Conclusion}

In summary, we employ all-atom molecular simulations to investigate the structural characteristics of PNIPAM and its co-polymers with PS in an aqueous environment, below and above the LCST of PNIPAM. In particular, we simulate single chains of 30-mer PNIPAM and PS at $275 \mathrm{~K}$ and $325 \mathrm{~K}$. The co-polymer systems investigated consist of PNIPAM-PS and PSPNIPAM-PS block co-polymers where each polymer chain consisted of 30 monomer units. The structure analysis of the polymer chains is conducted with radius of gyration $\left(\mathrm{R}_{\mathrm{g}}\right)$, small angle neutron scattering (SANS) calculations. The results for PS 30-mer suggest that due to its hydrophobic nature PS remains in globule-like state both at $275 \mathrm{~K}$ and $325 \mathrm{~K}$. On the other hand, pure PNIPAM 30-mer and PNIPAM in its co-polymers with PS remain in coil-state at $275 \mathrm{~K}$ and exhibit a clear coil-to-globule transition above LCST. In addition, we find that with increase in number of PS units, coil-to-globule transition of PNIPAM is accelerated. PNIPAM with 0-mer, 30-mer, and 60-mer of PS units undergoes a coil-to-globule transition in $\sim 34, \sim 24$, and $\sim 14$ ns, respectively. The distribution of PS units near PNIPAM chains shows aggregation of PNIPAM and PS monomers above the LCST. 
The structure of proximal water is analyzed by calculating radial distribution function (RDF) of water with backbone of polymer chains. We find that the structure of water near PNIPAM backbone is significantly different at $275 \mathrm{~K}$ as compared to $325 \mathrm{~K}$, which is attributed to the coillike and globule-like states of PNIPAM, respectively. In the case of PNIPAM-co-PS copolymers we observe that the backbone of PNIPAM polymer chain dehydrated more with increase in number of PS units, suggesting that presence of PS units alters the interactions between PNIPAM and proximal water molecules. The RDF of backbone carbon atoms in the PS

30-mer and oxygen of water molecules shows no significant difference in the structure of water near the backbone of PS at $325 \mathrm{~K}$ and $275 \mathrm{~K}$. Overall, PNIPAM chains are more hydrated as compared to PS chains. Below the LCST of PNIPAM, the water molecules near hydrophobic groups of PNIPAM units reorient themselves to form a cage-like structure, while water molecules near hydrophilic groups form hydrogen bonds with PNIPAM chains. The extent and rate of dehydration of PNIPAM monomer units increases with rise in PS units above the LCST.

Stability of the atomically ordered first hydration shell around the polymer is evaluated by analysing the residence probability and hydrogen bonding characteristic of polymers and copolymers in water. $\mathrm{P}_{\text {res }}(\mathrm{t})$ decreases in presence of PS chains, which suggests that PNIPAMwater interaction weakens in presence of PS chains. Increase in the number of PS units in the system results in a higher decay rate of hydrogen bonds. In general, with an increase in the number of PS monomer units, water forms less stable hydrogen bond network with PNIPAM, corroborating that the presence of PS chains does influence the hydrogen bond interactions between water and polymer both below and above the LCST. Our results reveal that presence of hydrophobic groups weakens the PNIPAM-water interactions leading to faster collapse of PNIPAM chains above its LCST. This information is very crucial for polymer chemists as it provides a guide for synthesizing novel co-polymers with improved control and efficacy for a range of biological and non-biological applications.

\section{Acknowledgments}

The authors acknowledge the use of the Center for Nanoscale Materials, supported by the U. S. Department of Energy, Office of Science, Office of Basic Energy Sciences, under Contract No. DE-AC02-06CH11357, for this project. This research used resources of the National Energy 
Research Scientific Computing Center, which is supported by the Office of Science of the U.S. Department of Energy under Contract No. DE-AC02-05CH11231, and of the Argonne Leadership Computing Facility at Argonne National Laboratory, which is supported by the Office of Science of the U.S. Department of Energy under contract DE-AC02-06CH1 1357. 


\section{References}

(1) Schild, H. G. Poly ( N-Isopropylacrylamide ): Experiment, Theory and Application. Progress in Polymer Science 1992, 17, 163-249.

(2) Deshmukh, S. A.; Sankaranarayanan, S. K. R. S.; Suthar, K.; Mancini, D. C. Role of Solvation Dynamics and Local Ordering of Water in Inducing Conformational Transitions in poly(N -Isopropylacrylamide) Oligomers through the LCST. Journal of Physical Chemistry B 2012, 116, 2651-2663.

(3) Aseyev, V. O.; Tenhu, H.; Winnik, F. M. Temperature Dependence of the Colloidal Stability of Neutral Amphiphilic Polymers in Water. Advances in Polymer Science 2006, 196, 185.

(4) Deshmukh, S.; Mooney, D. A.; MacElroy, J. M. D. Molecular Simulation Study of the Effect of Cross-Linker on the Properties of poly(N-Isopropyl Acrylamide) Hydrogel. Molecular Simulation 2011, 37, 846-854.

(5) Liu, H.; Avoce, D.; Song, Z.; Zhu, X.. N-Isopropylacrylamide Copolymers with Acrylamide and Methacrylamide Derivatives of Cholic Acid: Synthesis and Characterization. Macromolecular Rapid Communications 2001, 22, 675-680.

(6) Ilmain, F.; Tanaka, T.; Kokufuta, E. Volume Transition in a Gel Driven by Hydrogen Bonding. Nature 1991, 349, 400-401.

(7) Hugouvieux, V.; Axelos, M. a V; Kolb, M. Amphiphilic Multiblock Copolymers : From Intramolecular Pearl Necklace to Layered Structures Amphiphilic Multiblock Copolymers: From Intramolecular Pearl Necklace to Layered Structures. Macromolecules, 2009, 392-400.

(8) Bivigou-Koumba, A. M.; Kristen, J.; Laschewsky, A.; Müller-Buschbaum, P.; Papadakis, C. M. Synthesis of Symmetrical Triblock Copolymers of Styrene and N-Isopropylacrylamide Using Bifunctional Bis(trithiocarbonate)s as RAFT Agents. Macromolecular Chemistry and Physics 2009, 210, 565-578.

(9) Du, H.; Wickramasinghe, R.; Qian, X. Effects of Salt on the Lower Critical Solution Temperature of Poly (N-Isopropylacrylamide). The journal of physical chemistry. B 2010, 114, $16594-16604$.

(10) Abbott, L. J.; Tucker, A. K.; Stevens, M. J. Single Chain Structure of a Poly( $N$ Isopropylacrylamide) Surfactant in Water. The Journal of Physical Chemistry B 2015, 119, $3837-3845$. 
(11) Deshmukh, S.; Mooney, D. a.; McDermott, T.; Kulkarni, S.; Don MacElroy, J. M. Molecular Modeling of Thermo-Responsive Hydrogels: Observation of Lower Critical Solution Temperature. Soft Matter 2009, 5, 1514.

(12) Deshmukh, S. A.; Li, Z.; Kamath, G.; Suthar, K. J.; Sankaranarayanan, S. K. R. S.; Mancini, D. C. Atomistic Insights into Solvation Dynamics and Conformational Transformation in Thermo-Sensitive and Non-Thermo-Sensitive Oligomers. Polymer (United Kingdom) 2013, $54,210-222$.

(13) Deshmukh, S. A.; Kamath, G.; Suthar, K. J.; Mancini, D. C.; Sankaranarayanan, S. K. R. S. Non-Equilibrium Effects Evidenced by Vibrational Spectra during the Coil-to-Globule Transition in poly(N-Isopropylacrylamide) Subjected to an Ultrafast Heating-cooling Cycle. Soft Matter 2014, 10, 1462-1480.

(14) Deshmukh, S. A.; Sankaranarayanan, S. K. R. S.; Mancini, D. C. Vibrational Spectra of Proximal Water in a Thermo-Sensitive Polymer Undergoing Conformational Transition across the Lower Critical Solution Temperature. Journal of Physical Chemistry B 2012, 116, 55015515.

(15) Deshmukh, S. A.; Sankaranarayanan, S. K. R. S.; Mancini, D. C. Atomic Scale Characterization of the Conformational Dynamics of a Thermo-Sensitive and a Non-ThermoSensitive Oligomer Using Vibrational Spectra Obtained from Molecular Dynamics. Polymer 2012, 53, 1306-1320.

(16) Longhi, G.; Lebon, F.; Abbate, S.; Fornili, S. L. Molecular Dynamics Simulation of a Model Oligomer for poly(N-Isopropylamide) in Water. Chemical Physics Letters 2004, 386, $123-127$.

(17) Walter, J.; Ermatchkov, V.; Vrabec, J.; Hasse, H. Molecular Dynamics and Experimental Study of Conformation Change of poly(N-Isopropylacrylamide) Hydrogels in Water. Fluid Phase Equilibria 2010, 296, 164-172.

(18) Gangemi, F.; Longhi, G.; Abbaten, S.; Lebon, F.; Cordone, R.; Ghilardi, G. P.; Fornili, S. L. Molecular Dynamics Simulation of Aqueous Solutions of 26-Unit Segments of p(NIPAAm) and of p(NIPAAm) "doped" with Amino Acid Based Comonomers. Journal of Physical Chemistry B 2008, 112, 11896-11906. 
(19) Du, H. The Effects of Salt on the Lower Critical Solution Temperatures of poly(NIsopropylacrylamide) and Its Copolymer Studied from Molecular Dynamics Simulations. The Journal of Physical Chemistry B 2010 114, 16594-16604.

(20) Kamath, G.; Deshmukh, S. A; Baker, G. a; Mancini, D. C.; Sankaranarayanan, S. K. R. S. Thermodynamic Considerations for Solubility and Conformational Transitions of Poly-NIsopropyl-Acrylamide. Physical chemistry chemical physics : PCCP 2013, 15, 12667-12673.

(21) Pareek, P.; Adler, H. J. P.; Kuckling, D. Tuning the Swelling Behavior of Chemisorbed Thin PNIPAAm Hydrogel Layers by N,N-Dimethyl Acrylamide Content. Progress in Colloid and Polymer Science 2006, 132, 145-151.

(22) Tokarev, I.; Minko, S. Stimuli-Responsive Hydrogel Thin Films. Soft Matter 2009, 5, 511.

(23) Léonforte, F.; Müller, M. Poly( $N$-Isopropylacrylamide)-Based Mixed Brushes: A Computer Simulation Study. ACS Applied Materials \& Interfaces 2015, 7, 12450-12462.

(24) Gammas, S.; Suzuki, K.; Sone, C.; Sakurai, Y.; Kataoka, K.; Okano, T. ThermoResponsive Polymer Nanoparticles with a Core-Shell Micelle Structure as Site-Specific Drug Carriers. Journal of Controlled Release 1997, 48, 157-164.

(25) Wei, H.; Zhang, X.-Z.; Zhou, Y.; Cheng, S.-X.; Zhuo, R.-X. Self-Assembled Thermoresponsive Micelles of poly(N-Isopropylacrylamide-B-Methyl Methacrylate). Biomaterials 2006, 27, 2028-2034.

(26) Rzaev, Z. M. O.; Dinçer, S.; Pişkin, E. Functional Copolymers of N-Isopropylacrylamide for Bioengineering Applications. Progress in Polymer Science 2007, 32, 534-595.

(27) Yamato, M.; Utsumi, M.; Kushida, a; Konno, C.; Kikuchi, a; Okano, T. ThermoResponsive Culture Dishes Allow the Intact Harvest of Multilayered Keratinocyte Sheets without Dispase by Reducing Temperature. Tissue engineering 2001, 7, 473-480.

(28) Arvidsson, P.; Ivanov, a. E.; Galaev, I. Y.; Mattiasson, B. Polymer versus Monomer as Displacer in Immobilized Metal Affinity Chromatography. Journal of Chromatography B: Biomedical Sciences and Applications 2001, 753, 279-285.

(29) Callow, M. E.; Callow, J. a.; Ista, L. K.; Coleman, S. E.; Nolasco, a. C.; Lopez, G. P. Use of Self-Assembled Monolayers of Different Wettabilities to Study Surface Selection and Primary Adhesion Processes of Green Algal (Enteromorpha) Zoospores. Applied and Environmental Microbiology 2000, 66, 3249-3254. 
(30) Schacher, F. H.; Rupar, P. a.; Manners, I. Functional Block Copolymers: Nanostructured Materials with Emerging Applications. Angewandte Chemie - International Edition 2012, 51, $7898-7921$.

(31) Chung, J. E.; Yokoyama, M.; Okano, T. Inner Core Segment Design for Drug Delivery Control of Thermo-Responsive Polymeric Micelles. Journal of Controlled Release 2000, 65, 93103.

(32) Du, H.; Qian, X. Molecular Dynamics Simulations of PNIPAM-Co-PEGMA Copolymer Hydrophilic to Hydrophobic Transition in $\mathrm{NaCl}$ Solution. Journal of Polymer Science, Part B: Polymer Physics 2011, 49, 1112-1122.

(33) Shinde, V. S.; Girme, M. R.; Pawar, V. U. Thermoresponsive Polystyrene-B-poly(NIsopropylacrylamide) Copolymers by Atom Transfer Radical Polymerization. Indian Journal of Chemistry 2011, 50, 781-787.

(34) Nuopponen, M.; Ojala, J.; Tenhu, H. Aggregation Behaviour of Well Defined Amphiphilic Diblock Copolymers with poly(N-Isopropylacrylamide) and Hydrophobic Blocks. Polymer 2004, 45, 3643-3650.

(35) Chung, Z. E.; Yokoyama, M.; Suzuki, K.; Aoyagi, T.; Sakurai, Y.; Okano, T. Reversibly Thermo-Responsive Alkyl-Terminated poly(Nisopropylacrylamide) Core-Shell Micellar Structures. Colloids and Surfaces B: Biointerfaces 1997, 9, 37-48.

(36) Takei, Y. G.; Aoki, T.; Sanui, K.; Ogata, N.; Okano, T.; Sakurai, Y. TemperatureResponsive Bioconjugates. 2. Molecular Design for Temperature-Modulated Bioseparations. Bioconjugate Chem. 1993, 4, 341-346.

(37) Taylor, L. D.; Cerankowski, L. D. Preparation of Films Exhibiting a Balanced Temperature Dependence to Permeation by Aqueous Solutions-a Study of Lower Consolute Behavior. Journal of Polymer Science: Polymer Chemistry Edition 1975, 13, 2551-2570.

(38) Yoshida, R.; Sakai, K.; Okano, T.; Sakurai, Y. Modulating the Phase Transition Temperature and Thermosensitivity in N-Isopropylacrylamide Copolymer Gels. Journal of Biomaterials Science, Polymer Edition 1995, 6, 585-598.

(39) Suito, Y.; Isobe, Y.; Habaue, S.; Okamoto, Y. Isotactic-Specific Radical Polymerization of Methacrylamides in the Presence of Lewis Acids. J. Polym. Sci. A Polym. Chem., 40, pp. 2496-2500. 
(40) Ray, B.; Okamoto, Y; Kamigaito, M.; Sawamoto, M; Seno, K.; Kanaoka, S.; Aoshima, S. Effect of Tacticity of Poly ( $\mathrm{N}$-isopropylacrylamide ) on the Phase Separation Temperature of Its Aqueous Solutions. Polymer Journal, 2005, 37, 234-237.

(41) Katsumoto, Y.; Kubosaki, N. Tacticity Effects on the Phase Diagram for Poly ( N isopropylacrylamide ) in Water. Macromolecules, 2008, 41(15), 5955-5956.

(42) Chiessi, E.; Paradossi, G. Influence of Tacticity on Hydrophobicity of Poly (NIsopropylacrylamide): A Single Chain Molecular Dynamics Simulation Study. Journal of Physical Chemistry B, 2016, 120 (15), 3765-3776.

(43) Adelsberger, J.; Kulkami, A.; Jain, A.; Wang, W.; Bivigou-Koumba, A. M.; Busch, P.; Pipich, V.; Holderer, O.; Hellweg, T.; Laschewsky, Muller-Buschbaum, P; Papadakis, M., C. Thermoresponsive PS-B-PNIPAM-B-PS Micelles: Aggregation Behavior, Segmental Dynamics, and Thermal Response. Macromolecules 2010, 43, 2490-2501.

(44) Phillips, J. C.; Braun, R.; Wang, W.; Gumbart, J.; Tajkhorshid, E.; Villa, E.; Chipot, C.; Skeel, R. D.; Kalé, L.; Schulten, K. Scalable Molecular Dynamics with NAMD. Journal of Computational Chemistry 2005, 26, 1781-1802.

(45) Brooks, B. R.; Bruccoleri, R. E.; Olafson, B. D.; States, D. J.; Swaminathan, S.; Karplus, M. CHARMM: A Program for Macromolecular Energy, Minimization, and Dynamics Calculations. Journal of Computational Chemistry 1983, 4, 187-217.

(46) Sun, H.; Mumby, S. J.; Maple, J. R.; Hagler, A. T. An Ab Initio CFF93 All-Atom Force Field for Polycarbonates. Journal of the American Chemical Society 1994, 116, 2978-2987.

(47) Fritz, L.; Hofmann, D. Molecular Dynamics Simulations of the Transport of WaterEthanol Mixtures through Polydimethylsiloxane Membranes. Polymer 1997, 38, 1035-1045.

(48) Carrillo, J. M. Y.; Dobrynin, A. V. Detailed Molecular Dynamics Simulations of a Model NaPSS in Water. Journal of Physical Chemistry B 2010, 114, 9391-9399.

(49) Berndt, I.; Pedersen, J. S.; Richtering, W. Temperature-Sensitive Core-Shell Microgel Particles with Dense Shell. Angewandte Chemie - International Edition 2006, 45, 1737-1741.

(50) Grobelny, S.; Hofmann, C. H.; Erlkamp, M.; Plamper, F. a.; Richtering, W.; Winter, R. Conformational Changes upon High Pressure Induced Hydration of poly(NIsopropylacrylamide) Microgels. Soft Matter 2013, 9, 5862.

(51) D. Svergun. Crysol. Acta Crystallographica Section E: Structure Reports Online 1995, 60, 476-479. 
(52) Grant, T. D.; Luft, J. R.; Wolfley, J. R.; Tsuruta, H.; Martel, A.; Montelione, G. T.; Snell, E. H. Small Angle X-Ray Scattering as a Complementary Tool for High-Throughput Structural Studies. Biopolymers 2011, 95, 517-530.

(53) Deshmukh, S. a.; Sankaranarayanan, S. K. R. S. Atomic Scale Characterization of Interfacial Water near an Oxide Surface Using Molecular Dynamics Simulations. Physical Chemistry Chemical Physics 2012, 14, 15593.

(54) Tamai, Y.; Tanaka, H.; Nakanishi, K. Molecular Dynamics Study of Polymer-Water Interaction in Hydrogels. 2. Hydrogen-Bond Dynamics. Macromolecules 1996, 29, 6761-6769.

(55) Abbott, L. J.; Stevens, M. J. A Temperature-Dependent Coarse-Grained Model for the Thermoresponsive Polymer Poly (N-Isopropylacrylamide). The Journal Of Chemical Physics, 2015, 143, 244901.

(56) Essafi, W.; Spiteri, M. N.; Williams, C.; Boue, F. Hydrophobic Polyelectrolytes in Better Polar Solvent. Structure and Chain Conformation as Seen by SAXS and SANS. Macromolecules 2009, 42, 9568-9580.

(57) Svergun, D. I.; Richard, S.; Koch, M. H.; Sayers, Z.; Kuprin, S.; Zaccai, G. Protein Hydration in Solution: Experimental Observation by X-Ray and Neutron Scattering. Proceedings of the National Academy of Sciences of the United States of America 1998, 95, 2267-2272.

(58) Papagiannopoulos, A.; Zhao, J.; Zhang, G.; Pispas, S.; Radulescu, A. Thermoresponsive Aggregation of PS-PNIPAM-PS Triblock Copolymer: A Combined Study of Light Scattering and Small Angle Neutron Scattering. European Polymer Journal 2014, 56, 59-68.

(59) Dulle, M.; Jaber, S.; Rosenfeldt, S.; Radulescu, A.; Fo, S.; Mulvaney, P.; Karg, M. Plasmonic gold-poly(N-Isopropylacrylamide) Core-shell Colloids with Homogeneous Density Profiles: A Small Angle Scattering Study. Physical Chemistry Chemical Physics 2014, 17, 13541367.

(60) Li, X.; ShamsiJazeyi, H.; Pesek, S. L.; Agrawal, A.; Hammouda, B.; Verduzco, R. Thermoresponsive PNIPAAM Bottlebrush Polymers with Tailored Side-Chain Length and EndGroup Structure. Soft matter 2014, 10, 2008-2015.

(61) Chen, W. Synchronization of Ion Exchangers by an Oscillating Electric Field: Theory Synchronization of Ion Exchangers by an Oscillating Electric Field : Theory. Journal of Physical Chemistry B. 2008, 112, 10064-10070. 
(62) Pesek, S. L.; Li, X.; Hammouda, B.; Hong, K.; Verduzco, R. Small-Angle Neutron Scattering Analysis of Bottlebrush Polymers Prepared via Grafting-through Polymerization. Macromolecules 2013, 46, 6998-7005.

(63) Ono, Y.; Shikata, T. Hydration and Dynamic Behavior of Poly ( N-Isopropylacrylamide ) $\mathrm{S}$ in Aqueous Solution: A Sharp Phase Transition at the Lower Critical Solution Temperature. Macromolecules 2006, 10030-10031.

(64) Alaghemandi, M.; Spohr, E. A Molecular Dynamics Study of Poly (NIsopropylacrylamide) Endgrafted on a Model Cylindrical Pore Surface. RSC Advances, 2013, 3, 3638-3647. 


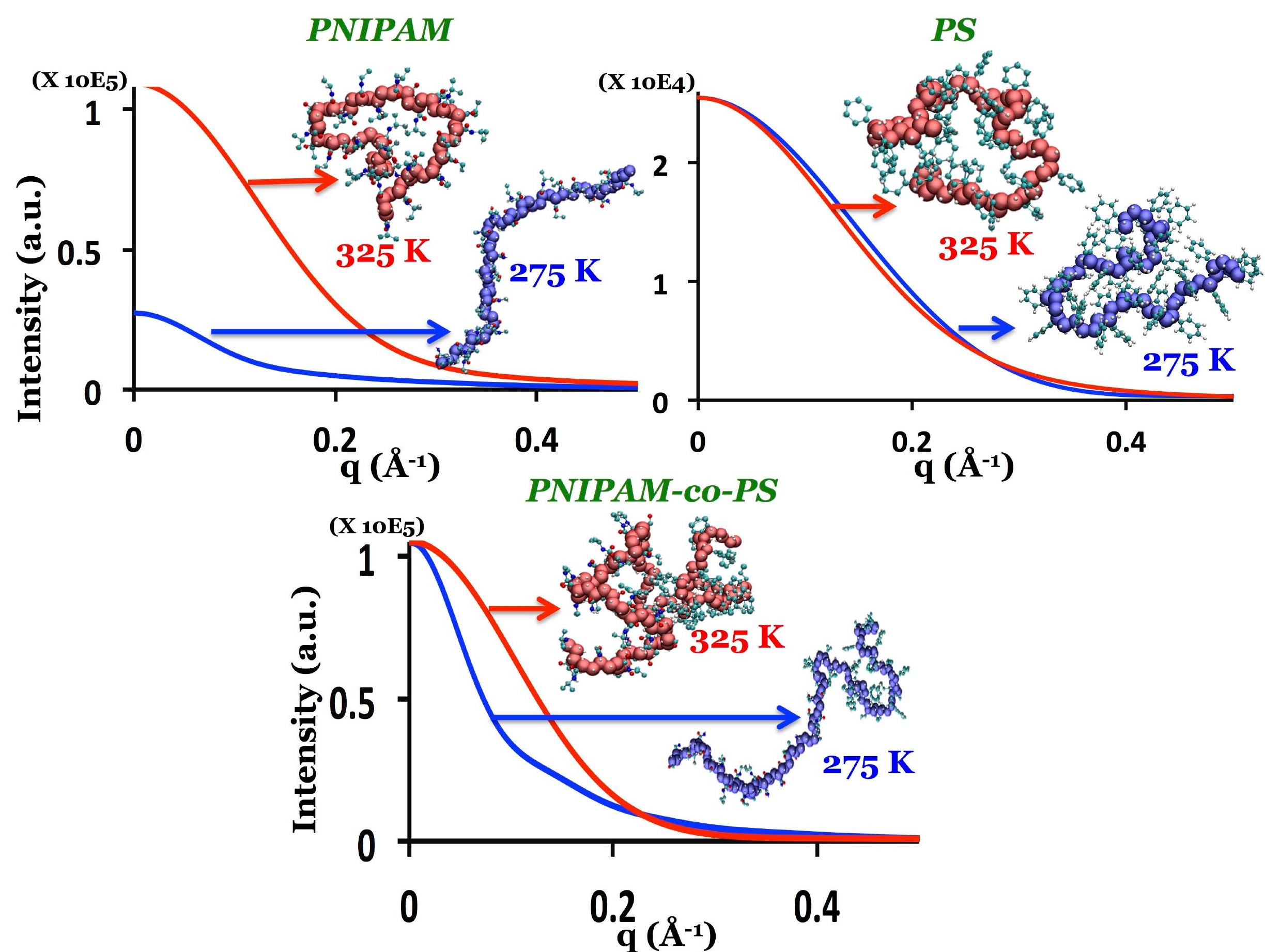

